\title{
A positive association between anxiety disorders and cannabis use or cannabis use disorders in the general population- a meta-analysis of 31 studies
}

\author{
Karina Karolina Kedzior ${ }^{*}$ and Lisa Tabata Laeber
}

\begin{abstract}
Background: The aim of the current study was to investigate the association between anxiety and cannabis use/ cannabis use disorders in the general population.

Methods: A total of $N=267$ studies were identified from a systematic literature search (any time- March 2013) of Medline and PsycInfo databases, and a hand search. The results of 31 studies (with prospective cohort or cross-sectional designs using non-institutionalised cases) were analysed using a random-effects meta-analysis with the inverse variance weights. Lifetime or past 12-month cannabis use, anxiety symptoms, and cannabis use disorders (CUD; dependence and/or abuse/harmful use) were classified according to DSM/ICD criteria or scores on standardised scales.

Results: There was a small positive association between anxiety and either cannabis use $(O R=1.24,95 \% \mathrm{Cl}$ : 1.06-1.45, $p=.006 ; N=15$ studies) or CUD (OR=1.68,95\% Cl: 1.23-2.31, $p=.001 ; N=13$ studies), and between comorbid anxiety + depression and cannabis use $(O R=1.68,95 \% \mathrm{Cl}: 1.17-2.40, p=.004 ; N=5$ studies). The positive associations between anxiety and cannabis use (or CUD) were present in subgroups of studies with ORs adjusted for possible confounders (substance use, psychiatric illness, demographics) and in studies with clinical diagnoses of anxiety. Cannabis use at baseline was significantly associated with anxiety at follow-up in $N=5$ studies adjusted for confounders $(O R=1.28,95 \% \mathrm{Cl}: 1.06-1.54, p=.01)$. The opposite relationship was investigated in only one study. There was little evidence for publication bias.
\end{abstract}

Conclusion: Anxiety is positively associated with cannabis use or CUD in cohorts drawn from some 112,000 non-institutionalised members of the general population of 10 countries.

Keywords: Anxiety disorders, Cannabis use, Cannabis use disorders (dependence, abuse), Systematic review, Meta-analysis

\section{Background}

The prevalence of affective and anxiety disorders is high, particularly in the western world. According to the annual Health Report 2012 of the second largest national health insurer in Germany (Techniker Krankenkasse), the absence from work due to a diagnosed psychiatric disorder had increased by about $70 \%$ from $2000-2012$ among clients insured with the company [1]. Next to depression, the most frequent of these diagnoses were different subtypes of anxiety disorders. Although such an extreme increase in

\footnotetext{
* Correspondence: kkedzior@graduate.uwa.edu.au

School of Humanities and Social Sciences, Jacobs University Bremen, Campus Ring 1, 28759 Bremen, Germany
}

\section{Ciomed Central}

(c) 2014 Kedzior and Laeber; licensee BioMed Central Ltd. This is an Open Access article distributed under the terms of the Creative Commons Attribution License (http://creativecommons.org/licenses/by/2.0), which permits unrestricted use, distribution, and reproduction in any medium, provided the original work is properly credited. prevalence of psychiatric illness was not confirmed based on the national survey data in Germany and other European Union (EU) countries over the same period of time [2], it is clear that psychiatric illnesses are common worldwide and substantially contribute to an overall disease burden. According to nationally-representative samples of adults, particularly the anxiety disorders occur frequently with a lifetime prevalence of $<10 \%$ in China, Israel, Nigeria, and Japan, between 10-20\% in the EU, Ukraine, Mexico, South Africa, and Lebanon, and $>20 \%$ in France, New Zealand, Columbia, and the highest prevalence of $31 \%$ in the USA $[2,3]$. Not surprisingly, compared to older adults (65+ years of age), the high rates of anxiety disorders are 
observed especially among the younger adults (18-34 years of age) during the challenging phase of life associated with establishment of professional career paths and long-term relationships/family [3]. Similarly to adults, the 12-month estimates show that anxiety disorders are also the most commonly occurring disorders in 13-17 year old adolescents in the USA [4]. Furthermore, the Australian data (from The National Survey of Mental Health and Wellbeing) suggest that more females (32\%) than males (20\%) experience anxiety disorders (especially the posttraumatic stress disorder, PTSD) in their lifetime [5].

After alcohol and nicotine, cannabis is the most widely consumed illicit substance [6,7] with an estimated 13.1 million dependent users worldwide in 2010 [8]. Interestingly, the highest estimated cumulative incidence of cannabis use (42\%) was observed in the two countries with the highest rates of anxiety disorders- the USA and New Zealand [6]. These findings suggest that anxiety and cannabis use might be related either directly or indirectly via common factors. However, the empirical evidence so far suggests that, among psychiatric disorders, cannabis use is most consistently associated only with psychosis [9]. According to meta-analyses, such an association is particularly strong in frequent users compared to ever users [10], early cannabis use increases the odds of later psychosis [11], cannabis use (independent of gender or heaviness of use) is related to an earlier onset of psychosis by about 3 years [12], and cannabis use disorders are observed in particularly younger, male, first-episode patients with schizophrenia [13]. In contrast, heavy or problematic cannabis use was only moderately related to depression outcomes $[10,14,15]$ and anxiety [16]. However, such associations were often eliminated after controlling for confounding factors, such as other substance use and/or other psychiatric comorbidity [16-18]. This result is not surprising because cannabis use is strongly associated with the use of other substances, especially nicotine [19], and nicotine use and dependence are related to some anxiety disorders, such as panic disorder and generalised anxiety disorder [20]. Similarly, clinical comorbidities frequently exist among different subtypes of anxiety disorders and depression [21] or alcohol use disorders [22]. Regardless of these possible confounders, a systematic, quantitative assessment of comorbidity between anxiety and cannabis use is needed because both conditions commonly occur in the general population, particularly during the most productive yet stressful lifephases (adolescent-middle age). If the two conditions coexist after controlling for confounders then this result might have some implications for clinical treatment and policy making. Specifically, the methods of detection and treatment of both conditions concurrently might need to be reassessed to provide effective patient care [23]. Such treatment could reduce any misdiagnoses because the acute effects of cannabis use resemble symptoms of anxiety disorders, such as panic attacks [16]. However, those seeking treatment for symptoms of anxiety may not disclose their cannabis use due to the illegal nature of this activity [14]. Furthermore, the link to anxiety could also be used as supplementary evidence for development of a rational cannabis policy that currently differs among countries worldwide [24].

To the best of our knowledge, only one systematic review so far attempted to quantify the association between cannabis use and anxiety disorders [10]. The authors listed the effect sizes based on $N=7$ studies using nationallyrepresentative cohorts and focusing on the association between 'cannabis exposure' and 'anxiety outcomes' (Figure six of the article). However, due to a high heterogeneity among these seven studies, their effect sizes were not combined quantitatively in a meta-analysis. The studies were not comparable because they utilised different classifications of anxiety disorders (either anxiety alone or comorbid anxiety and depression) and cannabis use (either use vs. no use or use with vs. without cannabis use disorders, such as dependence or abuse/harmful use). Therefore, it is not surprising that the effect sizes in the seven studies were highly inconsistent and did not suggest any general trend towards presence or absence of a relationship between anxiety and cannabis use [10].

The aim of the current study was to quantitatively assess the relationship between anxiety and cannabis use by means of a systematic literature review followed up by a quantitative meta-analysis. In contrast to Moore and colleagues [10], our aim was to include not only the large longitudinal studies but also smaller cross-sectional studies. The reasons for including such studies were to improve the statistical power of meta-analysis and to perform the analyses on subgroups of more homogenous studies. Specifically, our aim was to conduct the statistical metaanalysis on subgroups of studies based on similar classification of anxiety disorders (anxiety alone vs. comorbid anxiety and depression) and cannabis use (use vs. no use or use with vs. without cannabis use disorders). Finally, it was of interest to find out if an adequate volume of studies exists to test the direction of the main relationship. Specifically, our aim was to compute the odds for cannabis use at follow-up in cohorts with baseline anxiety and the odds for anxiety at follow-up in cohorts with baseline cannabis use.

It was hypothesised that a positive relationship exists between anxiety disorders and cannabis use (particularly with cannabis use disorders) based on the positive relationships between particularly heavy cannabis use and other psychiatric diagnoses (such as psychosis and depression). We expected that some evidence for the direction of such a relationship could emerge from the analysis of prospective studies. Specifically, we could establish which baseline 
condition (anxiety or cannabis use) would be related to higher odds of the other condition at follow-up. Furthermore, it was expected that the association between anxiety and cannabis use (or cannabis use disorders) would be lower (or even absent) in studies with effect sizes adjusted for confounding factors, such as other substance use, other psychiatric comorbidity, and demographics (age, gender), compared to studies with unadjusted effect sizes. Finally, it cannot be ruled out that the use of more advanced quantitative models to establish prevalence of comorbid conditions had some influence on the effect sizes reported in primary studies over time. Thus, we expected that there would be a negative relationship between effect sizes and the date of publication of studies (regardless of when the data were collected). Specifically, we hypothesised that the effect sizes would be adjusted for more factors using more complex statistical models (and thus be lower) in the newer than in the older studies.

\section{Methods}

Systematic literature search

A systematic literature search was conducted in March 2013, according to the steps depicted in Table 1.

A total of $N=267$ sources identified during the search (Table 1) were assessed and $N=218$ studies were excluded (the reasons for exclusion are summarised in the Additional file 1: Table S1).

\section{Study selection}

The summary of the study selection procedure and exclusion criteria are shown in the PRISMA flowchart [25], Figure 1.

Of $N=267$ sources, $N=49$ studies were retrieved and assessed in full-length. All these studies and, if applicable, the reasons for their exclusion are listed in the Additional file 1: Table S2. A total of $N=31$ studies were selected for inclusion in the final analysis. Studies were included if they reported:

(1) Data based on samples from a non-institutionalised, general population;

(2) Anxiety diagnoses (with or without comorbid depression), based on DSM/ICD diagnostic criteria, and/or anxiety severity score, based on standardised scales, in cannabis users/non-users or cannabis users with/without cannabis use disorder (CUD). CUD was defined as dependence and/or abuse/ harmful use according to DSM/ICD criteria;

(3) Cannabis use/no use (or cannabis use with/without CUD) in cases with anxiety/no anxiety;

(4) Odds ratios, ORs, and their $95 \%$ confidence intervals, 95\% CI (or sufficient information to compute these values), for

- anxiety/no anxiety in cannabis users/non-users or cannabis users with/without CUD or

- cannabis use/no use or cannabis use with/ without CUD in cases with anxiety/no anxiety;

(5) Data sufficient to compute other effect sizes, that were later converted into $O R$, such as the standardised mean difference (Cohen's $d$ ), based on means, $S D$, and $N$ of severity of anxiety scores in cannabis users/non-users (or cannabis users with/ without CUD).

Studies were excluded if they:

(1) Did not report data from healthy non-users;

(2) Reported data from samples seeking treatment for CUD and/or with a high comorbidity of othersubstance use and/or other concurrent psychiatric disorders than anxiety and depression;

(3) Reported inadequate data to compute any effect size (unless the authors provided additional data).

\section{Data extraction}

The data from $N=31$ studies were extracted/doublechecked for consistency by both authors.

The characteristics of all $N=31$ studies and the effect size data from these studies are listed in Tables 2 and 3. To maintain a high consistency of results the following data extraction rules were used:

(1) Only the most conservative estimates of ORs were extracted (i.e. the ORs adjusted for as many confounders as possible). If data based on the same cases were presented in more than one study then

Table 1 Details of the systematic literature search (all searches conducted in English with no language restrictions)

\begin{tabular}{|c|c|c|}
\hline Search & Search terms & Databases and timeframes \\
\hline Search $1 N=131$ & $\begin{array}{l}\text { Subject OR Title (cannabis or marijuana or marihuana) AND Subject OR Title ("affective disorder" } \\
\text { or "anxiety disorder" or anxiety) NOT (mouse or mice or rat or rats) }\end{array}$ & Psyclnfo (1806-March 2013) \\
\hline Search $2 N=168$ & $\begin{array}{l}\text { Subject OR Title (cannabis or marijuana or marihuana) AND Subject OR Title ("affective disorder" } \\
\text { or "anxiety disorder" or anxiety) AND Keyword (misus* or abus* or depend* or "harmful use" } \\
\text { or "harmful usage") NOT (mouse or mice or rat or rats) }\end{array}$ & Medline (1950-March 2013) \\
\hline $\boldsymbol{N}=256$ & Total $N$ from both searches excluding duplicates & \\
\hline
\end{tabular}

Note: Additional $N=11$ studies were obtained from other meta-analyses being conducted by the authors, hand-searches of bibliographies of retrieved sources, and from the bibliography of Moore et al. [10].

$N=$ number of sources identified during each search. 


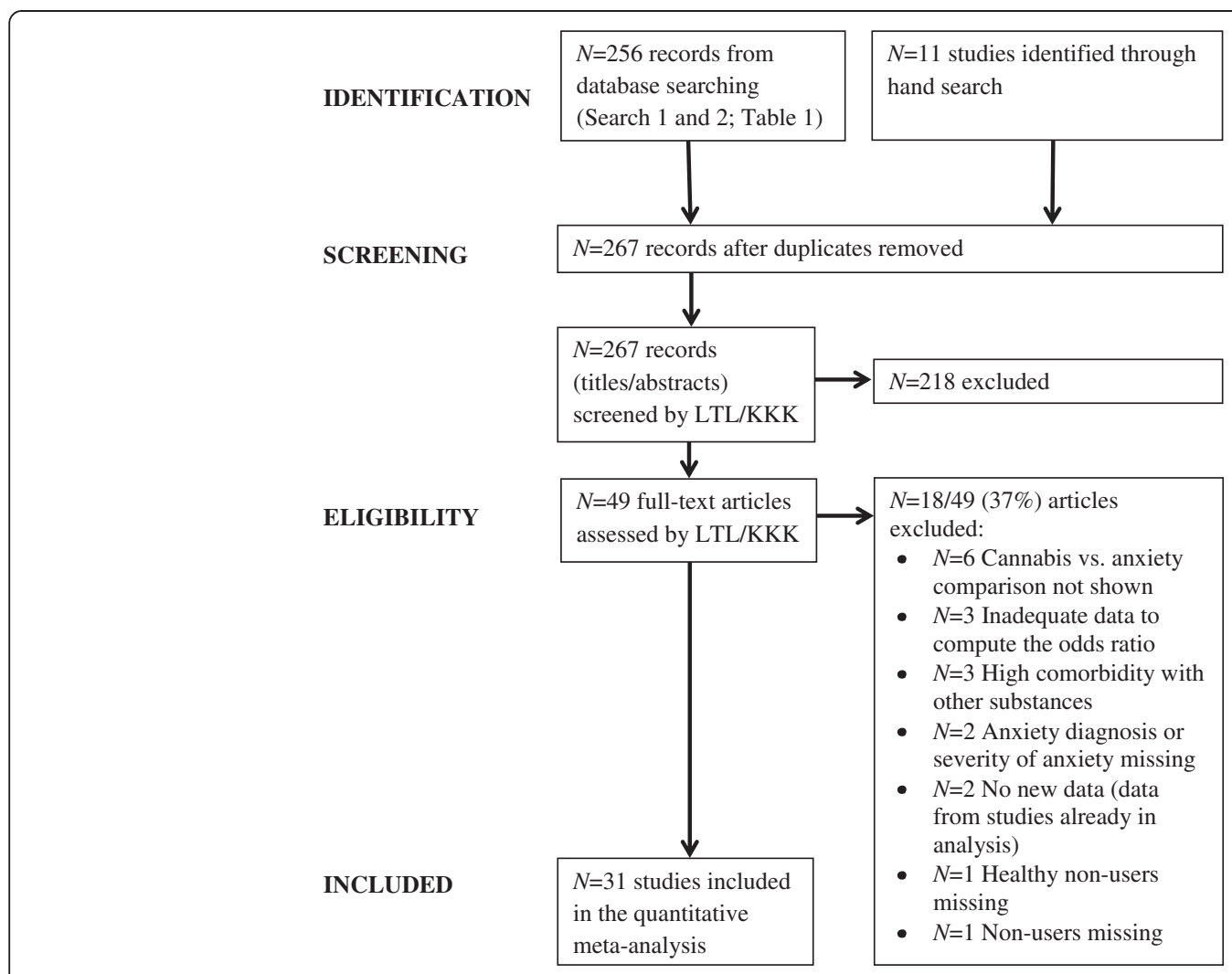

Figure 1 Selection of studies and exclusion criteria.

the study with more conservative ORs was selected for the final analysis;

(2) Data based on the longest possible time-spans (lifetime) were extracted;

(3) Data based on the heaviest use and/or cannabis dependence were extracted;

(4) Consistent estimates were extracted to combine ORs from different studies using data from the same cases (e.g. lifetime PTSD and lifetime panic disorder from two NCS-R studies, see Table 3);

(5) As many types of anxiety disorders as possible were included if ORs for individual anxiety disorders were reported separately for different groups of cases.

\section{Meta-analysis}

The mathematical details of meta-analysis used in the current study are explained in the Additional file 1. The analysis was conducted on the Comprehensive MetaAnalysis software (CMA 2.2; Biostat, USA) using the random-effects model [57]. This model was chosen because it was expected that only a random sample of all studies (published or unpublished) on the topic was located during the systematic literature search, that the effect sizes would differ due to methodological heterogeneity among studies in this analysis, and that the current results could be generalisable to a wider general population.
In the first step of the analysis, a common effect size, the odds ratio $(O R)$ and its $95 \%$ confidence interval (95\% CI), were either computed by the authors or extracted from the $N=31$ studies (Table 3 ). Subsequently, all effect sizes were weighted based on the inversevariance method also known as the method of moments or DerSimonian and Laird method [58]. According to this method the weight is defined as the inverse of the sum of within-study and between-study variance. Compared to larger studies, the smaller- $N$ studies usually have high variability of scores (high variance, low precision) and thus low weight. Consequently, such small studies have little influence on the overall mean weighted $O R$ and vice versa.

The overall mean weighted $O R$ was computed by dividing the sum of the product of weights and log ORs in each study by the sum of all weights. An $O R<1$ indicated that cannabis use (or CUD) is associated with lower prevalence of anxiety and vice versa. The magnitude of $O R$ was interpreted as follows [59]:

- $O R>1: 1.5$ (small), 2.5 (moderate), $\geq 4$ (large)

- $O R<1$ : 0.67 (small), 0.4 (moderate), $\leq 0.25$ (large)

- $O R=1$ and/or 95\% CI crossing the line of no effect $(O R=1)$ : no association between cannabis use (or CUD) and anxiety. 
Table $\mathbf{2}$ Characteristics of $\mathbf{N}=\mathbf{3 1}$ studies included in the current meta-analysis

\begin{tabular}{|c|c|c|c|c|c|c|c|c|c|c|}
\hline Study; Country & Design* & $\begin{array}{l}\text { Total } N(N \text { in } \\
\text { this study**) }\end{array}$ & Sample type & $\begin{array}{l}\text { Sampling } \\
\text { method }\end{array}$ & $\begin{array}{l}\text { Anxiety } \\
\text { assessment; } \\
\text { diagnostic } \\
\text { system }\end{array}$ & $\begin{array}{l}\text { Anxiety } \\
\text { diagnosis } \\
\text { (timeframe) }\end{array}$ & $\begin{array}{l}\text { Anxiety } \\
\text { prevalence }\end{array}$ & $\begin{array}{l}\text { Cannabis } \\
\text { assessment; } \\
\text { diagnostic } \\
\text { system }\end{array}$ & $\begin{array}{l}\text { Cannabis use; } \\
\text { CUD (timeframe) }\end{array}$ & $\begin{array}{l}\text { Cannabis use/ } \\
\text { CUD prevalence }\end{array}$ \\
\hline $\begin{array}{l}\text { Agosti et al., 2002; } \\
\text { NCS, USA [26] }\end{array}$ & Cross-sectional & 8098 & $\begin{array}{l}\text { General } \\
\text { population }\end{array}$ & Probability & CIDI DSM-III-R & AD (current) & - & CIDI DSM-III-R & $\begin{array}{l}\text { Use (past month); } \\
\text { CD (lifetime) }\end{array}$ & - \\
\hline $\begin{array}{l}\text { Beard et al., 2006; } \\
\text { NoRMHS, Australia [27] }\end{array}$ & Longitudinal & 9191 (1013) & $\begin{array}{l}\text { General } \\
\text { population }\end{array}$ & Random & CIDI ICD-10 & $\begin{array}{l}\mathrm{PD}, \mathrm{SP}, \mathrm{OCD} \\
\text { (at T1) }\end{array}$ & 15\% (at T1) & CIDI ICD-10 & CUD (at T0) & CUD 3\% (at T0) \\
\hline $\begin{array}{l}\text { Brook et al., 1998; } \\
\text { Upstate NY, USA [28] }\end{array}$ & Longitudinal & $\begin{array}{l}975(\mathrm{~T} 3=745 \\
\mathrm{T} 4=698)\end{array}$ & $\begin{array}{l}\text { Adolescents- } \\
\text { adults }\end{array}$ & Random & DISC DSM-III-R & $\begin{array}{l}\text { SA, OAD, SAD } \\
\text { (at T4) }\end{array}$ & - & DISC DSM-III-R & $\begin{array}{l}\text { Never- } \geq \text { weekly } \\
\text { (at T3) }\end{array}$ & $\begin{array}{l}\text { Use (at T3): 56\% } \\
\text { never 9\% } \geq \\
\text { weekly }\end{array}$ \\
\hline $\begin{array}{l}\text { Brook et al., 2001; } \\
\text { Colombia [29] }\end{array}$ & Longitudinal & 2226 & Adolescents & Random & $\mathrm{HSC}$ & $\mathrm{AD}$ (at T1, T2) & - & Interview & $\begin{array}{l}\text { Non-regular- regular } \\
\text { ( } \geq \text { monthly; lifetime) }\end{array}$ & - \\
\hline $\begin{array}{l}\text { Buckner et al., 2008; } \\
\text { Oregon, USA [30] }\end{array}$ & Longitudinal & $\begin{array}{l}\mathrm{T} 1=1709 \\
\mathrm{~T} 4=816\end{array}$ & $\begin{array}{l}\text { High school } \\
\text { students }\end{array}$ & Random & K-SADS DSM-III-R & SAD (at T1) & $2 \%$ (at T1) & $\begin{array}{l}\text { LIFE, SCID-I/ } \\
\text { NP DSM-IV }\end{array}$ & CD (at T4) & CD 6\% (at T4) \\
\hline $\begin{array}{l}\text { Buckner \& Schmidt, } \\
\text { 2008; USA [31] }\end{array}$ & Cross-sectional & 337 (214) & $\begin{array}{l}\text { Undergraduate } \\
\text { students }\end{array}$ & Random & SIAS & $\begin{array}{l}\text { Equivalent to } \\
\text { SAD }\end{array}$ & $\begin{array}{l}19 \% \text { (scores } \\
\text { in clinical } \\
\text { range) }\end{array}$ & Questionnaire & $\begin{array}{l}\text { Never-frequent } \\
\text { ( } \geq \text { weekly; lifetime) }\end{array}$ & $\begin{array}{l}31 \% \text { never } 32 \% \\
\text { frequent }\end{array}$ \\
\hline $\begin{array}{l}\text { Buckner et al., 2012; } \\
\text { USA [32] }\end{array}$ & Cross-sectional & $343(200)$ & $\begin{array}{l}\text { Adult tobacco } \\
\text { smokers }\end{array}$ & Random & SIAS & $\begin{array}{l}\text { Equivalent to } \\
\text { SAD }\end{array}$ & - & Questionnaire & $\begin{array}{l}\text { Never- current } \\
\text { (daily; past month) }\end{array}$ & $\begin{array}{l}\text { 19\% never } 39 \% \\
\text { current use; } 13 \% \\
\text { current daily }\end{array}$ \\
\hline $\begin{array}{l}\text { Cascone et al., 2011; } \\
\text { Switzerland [33] }\end{array}$ & Cross-sectional & 110 & $\begin{array}{l}\text { Adolescents in } \\
\text { schools/ } \\
\text { psycho- } \\
\text { educational } \\
\text { unit }\end{array}$ & Convenience & STAI-Y B & Trait anxiety & - & $\begin{array}{l}\text { ADAD DSM- } \\
\text { IV-TR }\end{array}$ & $\begin{array}{l}\text { Use (past month) } \\
\text { CD (past } 12 \\
\text { months) }\end{array}$ & $\begin{array}{l}\text { Past month: No } \\
\text { CD: } 94 \% \text { none } \\
\text { CD: } 66 \% \text { daily }\end{array}$ \\
\hline $\begin{array}{l}\text { Chabrol et al., 2005; } \\
\text { France [34] }\end{array}$ & Cross-sectional & 212 & $\begin{array}{l}\text { High school } \\
\text { and college } \\
\text { students }\end{array}$ & Random & STAI A & State anxiety & - & $\begin{array}{l}\text { Questionnaire } \\
\text { DSM-IV }\end{array}$ & $\begin{array}{l}\text { Past } 6 \text { months: } \\
\text { None- > daily; CD }\end{array}$ & $\begin{array}{l}46 \% \text { none } 23 \%> \\
\text { daily }\end{array}$ \\
\hline $\begin{array}{l}\text { Chabrol et al., 2008; } \\
\text { France [35] }\end{array}$ & Cross-sectional & 248 & $\begin{array}{l}\text { High school } \\
\text { students }\end{array}$ & Random & STAI A & State anxiety & - & Interview & $\begin{array}{l}\text { None- use } \geq 1 \times \\
\text { (past } 6 \text { months) }\end{array}$ & $\begin{array}{l}76 \% \text { none } 24 \% \\
\text { users }\end{array}$ \\
\hline $\begin{array}{l}\text { Cheung et al., 2010; } \\
\text { CAMH, Canada [36] }\end{array}$ & $\begin{array}{l}\text { Cross-sectional } \\
\text { cycles of } 2001- \\
2006 \text { survey }\end{array}$ & 14531 (13478) & $\begin{array}{l}\text { General } \\
\text { population }\end{array}$ & Probability & $\begin{array}{l}\text { GHQ12 } \geq 4 / 12 \\
\text { symptoms }\end{array}$ & $\begin{array}{l}\text { AMD (past } \\
12 \text { months) }\end{array}$ & $\begin{array}{l}9 \% \text { (past } \\
12 \text { months) }\end{array}$ & Interview & $\begin{array}{l}\text { None- daily (past } \\
12 \text { months) }\end{array}$ & - \\
\hline $\begin{array}{l}\text { Cougle et al., 2011; } \\
\text { NCS-R, USA [37] }\end{array}$ & Cross-sectional & 5672 & $\begin{array}{l}\text { General } \\
\text { population }\end{array}$ & $\begin{array}{l}\text { Stratified } \\
\text { probability }\end{array}$ & CIDI DSM-IV & PTSD (lifetime) & 7\% (lifetime) & $\mathrm{CIDI}$ & $\begin{array}{l}\text { Never- use } \geq 1 \times \\
\text { (lifetime) }\end{array}$ & $\begin{array}{l}42 \% \text { users } \\
\text { (lifetime) }\end{array}$ \\
\hline $\begin{array}{l}\text { Crum et al., 1993; } \\
\text { ECA, USA [38] }\end{array}$ & Longitudinal & $18572(577)$ & $\begin{array}{l}\text { General } \\
\text { population }\end{array}$ & Probability & DIS DSM-III & $\begin{array}{l}\text { OCD (past } \\
12 \text { months) }\end{array}$ & $\begin{array}{l}1 \% \text { (past } \\
12 \text { months) }\end{array}$ & DIS & $\begin{array}{l}\text { No use- use } \geq 6 \times \\
\text { (past } 12 \text { months) }\end{array}$ & $\begin{array}{l}84 \% \text { no drug use } \\
9 \% \text { cannabis }\end{array}$ \\
\hline $\begin{array}{l}\text { Degenhardt et al., } \\
\text { 2001; NSMHWB, } \\
\text { Australia [39] }\end{array}$ & Cross-sectional & 10641 & $\begin{array}{l}\text { General } \\
\text { population }\end{array}$ & $\begin{array}{l}\text { Stratified } \\
\text { random }\end{array}$ & CIDI DSM-IV & $\begin{array}{l}\mathrm{SAD}, \mathrm{AP}, \mathrm{PD}, \\
\mathrm{GAD}, \mathrm{OCD}, \mathrm{PTSD} \\
\text { (past } 12 \text { months) }\end{array}$ & $\begin{array}{l}6 \% \text { (past } \\
12 \text { months) }\end{array}$ & CIDI DSM-IV & $\begin{array}{l}\text { Past } 12 \text { months: } \\
\text { No use- use } \geq 5 \times \text {; } \\
\text { CD }\end{array}$ & $\begin{array}{l}\text { Past } 12 \text { months: } \\
5 \% \text { users } 2 \% \text { CD }\end{array}$ \\
\hline $\begin{array}{l}\text { Degenhardt et al., } \\
\text { 2010; VAHCS, } \\
\text { Australia [40] }\end{array}$ & Longitudinal & $\begin{array}{l}1943(1520, \\
\text { wave } 1-8)\end{array}$ & $\begin{array}{l}\text { High school } \\
\text { students }\end{array}$ & $\begin{array}{l}\text { Stratified } \\
\text { random }\end{array}$ & $\begin{array}{l}\text { GHQ12 > } 2 \\
\text { symptoms (at 24) }\end{array}$ & AMD (at 24) & $21 \%$ (at 24) & Interview & $\begin{array}{l}\text { None- weekly+ } \\
\text { (past } 6 \text { months at } \\
15-17 \text { ) }\end{array}$ & $\begin{array}{l}34 \% \text { users (at } \\
15-17 \text { ) }\end{array}$ \\
\hline
\end{tabular}


Table 2 Characteristics of $\boldsymbol{N}=\mathbf{3 1}$ studies included in the current meta-analysis (Continued)

\begin{tabular}{|c|c|c|c|c|c|c|c|c|c|c|}
\hline $\begin{array}{l}\text { Degenhardt et al., } \\
\text { 2013; VAHCS, } \\
\text { Australia [41] }\end{array}$ & Longitudinal & $\begin{array}{l}1943(1756, \\
\text { wave } 1-9)\end{array}$ & $\begin{array}{l}\text { High school } \\
\text { students }\end{array}$ & $\begin{array}{l}\text { Stratified } \\
\text { random }\end{array}$ & CIDI ICD-10 & $\begin{array}{l}\text { GAD, SAD, PD, } \\
\text { AP (past } 12 \\
\text { months at 29) }\end{array}$ & $11 \%$ (at 29) & CIDI ICD-10 & $\begin{array}{l}\text { None/<weekly- } \\
\text { weekly + (past } \\
6-12 \text { months at } \\
15-29) ; \text { CD (past } \\
12 \text { months at 29) }\end{array}$ & $\begin{array}{l}\text { Lifetime: } 67 \% \\
\text { none/ <weekly } \\
2 \% \text { weekly+ } 4 \% \\
\text { CD (at 29) }\end{array}$ \\
\hline $\begin{array}{l}\text { Fergusson et al., } \\
\text { 1996; CHDS, New } \\
\text { Zealand [42] }\end{array}$ & Longitudinal & $1265(927)$ & $\begin{array}{l}\text { Adolescents } \\
\text { (16 years) }\end{array}$ & Stratified & DISC/DIS DSM-III-R & $\begin{array}{l}\text { GAD, OAD, SA } \\
\text { (at 15-16) }\end{array}$ & $9 \%$ (at 15-16) & Interview & $\begin{array}{l}\text { None- use (past } 12 \\
\text { months at } 15-16 \text { ) }\end{array}$ & $\begin{array}{l}20 \% \text { users } \\
\text { (at } 15-16)\end{array}$ \\
\hline $\begin{array}{l}\text { Hayatbakhsh et al., } \\
\text { 2007; MUSP, } \\
\text { Australia [43] }\end{array}$ & Longitudinal & $7223(3157)$ & $\begin{array}{l}\text { Adult children } \\
\text { of mothers in } \\
\text { study }\end{array}$ & Convenience & $\begin{array}{l}\text { YASR (resembles } \\
\text { DSM-III-R) }\end{array}$ & AMD (at 21) & - & Interview & $\begin{array}{l}\text { Never used drugs- } \\
\text { frequent } \leq \text { daily } \\
\text { (past month) }\end{array}$ & $12 \%$ frequent \\
\hline $\begin{array}{l}\text { Lamers et al., 2006; } \\
\text { USA [44] }\end{array}$ & Cross-sectional & $41(30)$ & $\begin{array}{l}\text { General } \\
\text { population }\end{array}$ & Convenience & $\mathrm{BAl}$ & BAl scores & - & $\begin{array}{l}\text { Questionnaire } \\
\text { urine screen }\end{array}$ & $\begin{array}{l}\text { None (past } 12 \\
\text { months)- use } \\
\geq 10 \times \text { (lifetime) }\end{array}$ & $\begin{array}{l}50 \% \text { non-users } \\
50 \% \text { users }\end{array}$ \\
\hline $\begin{array}{l}\text { Low et al., 2008; } \\
\text { USA [45] }\end{array}$ & Cross-sectional & 632 & $\begin{array}{l}\text { Adolescents in } \\
\text { primary care }\end{array}$ & Convenience & PRIME-MD DSM-IV & $\begin{array}{l}\mathrm{PD}, \mathrm{GAD}, \mathrm{AD} \\
\text { (past 1-6 } \\
\text { months) }\end{array}$ & $\begin{array}{l}7 \% \text { (past 1-6 } \\
\text { months) }\end{array}$ & $\begin{array}{l}\text { PRIME-MD } \\
\text { DSM-IV }\end{array}$ & $\begin{array}{l}\text { CA (past } 6 \\
\text { months) }\end{array}$ & $6 \% \mathrm{CA}$ \\
\hline $\begin{array}{l}\text { Martins \& Gorelick, } \\
\text { 2011; NESARC, } \\
\text { USA [46] }\end{array}$ & Cross-sectional & 43093 & $\begin{array}{l}\text { General } \\
\text { population }\end{array}$ & $\begin{array}{l}\text { Stratified } \\
\text { random }\end{array}$ & AUDADIS DSM-IV & $\begin{array}{l}\text { PD, AP, SP, GAD } \\
\text { (lifetime) }\end{array}$ & 17\% (lifetime) & $\begin{array}{l}\text { AUDADIS } \\
\text { DSM-IV }\end{array}$ & CA + CD (lifetime) & - \\
\hline $\begin{array}{l}\text { McGee et al., 2000; } \\
\text { DMHDS, New } \\
\text { Zealand [47] }\end{array}$ & Longitudinal & $1037(891)$ & $\begin{array}{l}\text { Adolescents } \\
\text { (at 15) }\end{array}$ & Convenience & DISC DSM-III & $\begin{array}{l}\text { Internalising } \\
\text { disorders (AMD; } \\
\text { at 15) }\end{array}$ & $12 \%$ (at 15 ) & Interview & $\begin{array}{l}\text { None- use } \geq 1 \\
\text { (past } 12 \text { months) }\end{array}$ & $14 \%$ users (at 15 ) \\
\hline $\begin{array}{l}\text { NPMS, UK; appendix, } \\
\text { Moore et al., } 2007 \text { [10] }\end{array}$ & Longitudinal & 8580 (1578) & $\begin{array}{l}\text { Adults (at } \\
16-74 \text { ) }\end{array}$ & Random & CIS-R $\geq 12$ & AMD & $11 \%$ CIS-R $\geq 12$ & Interview & $\begin{array}{l}\text { Use: no/yes } \\
\text { (lifetime); CD } \\
\text { (past } 12 \text { months): } \\
\text { no/yes }\end{array}$ & $16 \%$ users $2 \%$ CD \\
\hline $\begin{array}{l}\text { Patton et al., 2002; } \\
\text { VAHCS, Australia [48] }\end{array}$ & Longitudinal & $\begin{array}{l}1943(1601, \\
\text { wave } 1-7)\end{array}$ & $\begin{array}{l}\text { High school } \\
\text { students }\end{array}$ & $\begin{array}{l}\text { Stratified } \\
\text { random }\end{array}$ & $\mathrm{CIS}-\mathrm{R} \geq 12$ at 21 & AMD (at 21) & $16 \%$ (at 21) & Interview & $\begin{array}{l}\text { None- < weekly } \\
\text { (past } 6 \text { months at } \\
15-17 \text { ) }\end{array}$ & $\begin{array}{l}59 \% \text { users } \\
\text { (lifetime) }\end{array}$ \\
\hline $\begin{array}{l}\text { Roberts et al., 2007; } \\
\text { TH2K, USA [49] }\end{array}$ & Cross-sectional & 4175 & Adolescents & Probability & DISC DSM-IV & $\begin{array}{l}\text { AP, GAD, PD, } \\
\text { SAD, PTSD (past } \\
12 \text { months) }\end{array}$ & $\begin{array}{l}7 \% \text { (past } \\
12 \text { months) }\end{array}$ & DISC DSM-IV & $\begin{array}{l}\text { CUD (past } 12 \\
\text { months) }\end{array}$ & $\begin{array}{l}\text { 3\% CUD (past } 12 \\
\text { months) }\end{array}$ \\
\hline $\begin{array}{l}\text { Swift et al., 2008; } \\
\text { VAHCS, Australia [50] }\end{array}$ & Longitudinal & $\begin{array}{l}1943(1520 \\
\text { wave } 1-8)\end{array}$ & $\begin{array}{l}\text { High school } \\
\text { students }\end{array}$ & $\begin{array}{l}\text { Stratified } \\
\text { random }\end{array}$ & CIS-R $>11$ & AMD (at 15-17) & - & $\begin{array}{l}\text { Interview, CIDI } \\
\text { DSM-IV }\end{array}$ & $\begin{array}{l}\text { Past } 12 \text { months at } \\
\text { 24: None- weekly+; } \\
\text { CD }\end{array}$ & $\begin{array}{l}28 \% \text { weekly+ at } \\
24 \text { who used at } \\
15-17\end{array}$ \\
\hline $\begin{array}{l}\text { van der Pol et al., 2013; } \\
\text { CanDep + NEMESIS-2, } \\
\text { Netherlands [51] }\end{array}$ & Cross-sectional & $\begin{array}{l}\text { 1324: D+: } 252 \\
\text { N2: } 1072\end{array}$ & $\begin{array}{l}\text { General } \\
\text { population/ } \\
\text { 'coffee shop' } \\
\text { users (18-30) }\end{array}$ & $\begin{array}{l}\text { Stratified } \\
\text { random; } \\
\text { convenience/ } \\
\text { chain-referral }\end{array}$ & CIDI DSM-IV & $\begin{array}{l}\text { SAD, PD, GAD, } \\
\text { AP (past } 12 \\
\text { months) }\end{array}$ & $\begin{array}{l}8 \% \text { (past } \\
12 \text { months) }\end{array}$ & CIDI DSM-IV & $\begin{array}{l}\text { No CD (group N2; } \\
\text { none or }<3 \times / \text { week } \\
\text { use)- } C D \text { ( } D+\text {; use } \\
\geq 3 \times / \text { week) (past } \\
12 \text { months) }\end{array}$ & $\begin{array}{l}16 \% \text { CD (past } 12 \\
\text { months) }\end{array}$ \\
\hline $\begin{array}{l}\text { Van Laar et al., 2007; } \\
\text { NEMESIS, } \\
\text { Netherlands [52] }\end{array}$ & Longitudinal & $\begin{array}{l}\text { T0: } 7076 \\
\text { T2: } 4848\end{array}$ & $\begin{array}{l}\text { General } \\
\text { population }\end{array}$ & Probability & CIDI DSM-III-R & $\begin{array}{l}\text { PD, AP, SAD, SP, } \\
\text { GAD, OCD (3- } \\
\text { year incidence, } \\
\text { T0-T2) }\end{array}$ & 6\% (at T0-T2) & CIDI DSM-III-R & $\begin{array}{l}\text { No use- use }>5 \times \\
\text { (lifetime at T0) }\end{array}$ & - \\
\hline
\end{tabular}


Table 2 Characteristics of $\boldsymbol{N}=\mathbf{3 1}$ studies included in the current meta-analysis (Continued)

\begin{tabular}{|c|c|c|c|c|c|c|c|c|c|c|}
\hline $\begin{array}{l}\text { Wittchen et al., 2007; } \\
\text { EDSP, Germany [53] }\end{array}$ & Longitudinal & $\begin{array}{l}\text { T0: } 1395 \text { T3: } \\
\text { 1019 (1310) }\end{array}$ & $\begin{array}{l}\text { General } \\
\text { population }\end{array}$ & Random & CIDI DSM-IV & $\begin{array}{l}\text { PD, GAD, AP, } \\
\text { SAD, SP, SA, } \\
\text { OCD, PTSD } \\
\text { (at TO) }\end{array}$ & $23 \%$ (at T0) & CIDI DSM-IV & $\begin{array}{l}\text { Use: no/yes } \\
\text { (lifetime); CUD } \\
\text { (lifetime) }\end{array}$ & $\begin{array}{l}\text { Lifetime: } 54 \% \text { use } \\
13 \% \text { CUD }\end{array}$ \\
\hline $\begin{array}{l}\text { Zvolensky et al., 2006; } \\
\text { CSHS, USA [54] }\end{array}$ & Cross-sectional & 4745 & $\begin{array}{l}\text { General } \\
\text { population }\end{array}$ & $\begin{array}{l}\text { Stratified } \\
\text { random }\end{array}$ & DIS DSM-IV-TR & PA (lifetime) & 6\% (lifetime) & DIS DSM-IV-TR & $\begin{array}{l}\text { Lifetime: No use- } \\
\text { use } \geq 5 \times ; C D\end{array}$ & $\begin{array}{l}\text { Lifetime: } 25 \% \\
\text { users 1\% CD }\end{array}$ \\
\hline $\begin{array}{l}\text { Zvolensky et al., 2010; } \\
\text { NCS-R, USA [55] }\end{array}$ & Cross-sectional & 5672 & $\begin{array}{l}\text { General } \\
\text { population }\end{array}$ & $\begin{array}{l}\text { Stratified } \\
\text { probability }\end{array}$ & CIDI DSM-IV & PD (lifetime) & 6\% (lifetime) & $C|D|$ & $\begin{array}{l}\text { None- use } \geq 1 \times \\
\text { (lifetime) }\end{array}$ & $\begin{array}{l}42 \% \text { users } \\
\text { (lifetime) }\end{array}$ \\
\hline
\end{tabular}

Notes: All studies included males and females of any race. Abbreviations: $A D=$ anxiety disorder; $A D A D=$ Adolescent Drug Abuse Diagnosis (based on Addiction Severity Index); $A M D=$ anxiety + depression; $\mathrm{AP}=$ agoraphobia; AUDADIS = Alcohol Use Disorders and Associated Disabilities Interview Schedule; BAI = Beck Anxiety Inventory; $C A=$ cannabis abuse; $C A M H=C e n t r e$ for Addiction and Mental Health Monitor survey, Canada; CanDep = the Dutch Cannabis Dependence Study, Netherlands; CD = cannabis dependence; CHDS = Christchurch Health and Development Study, New Zealand; CIDI = Composite International Diagnostic Interview; CIS-R=Clinical Interview Schedule- Revised; CSHS = Colorado Social Health Survey, USA; CUD = cannabis use disorder (abuse/harmful use and/or dependence); $D+=$ frequent cannabis users with dependence in CanDep study; DIS = Diagnostic Interview Schedule; DISC = Diagnostic Interview Schedule for Children; DMHDS = Dunedin Multidisciplinary Health and Development Study, Dunedin, New Zealand; ECA = Epidemiological Catchment Area program, USA; EDSP = Early Developmental Stages of Psychopathology study, Germany; GAD = generalized anxiety disorder; GHQ-12= General Health Questionnaire (12 items); HSC = Hopkins Symptom Checklist; K-SADS = Schedule for Affective Disorders and Schizophrenia for School-Age Children; LIFE = Longitudinal Interval Follow-up Evaluation; MUSP = Mater University Study of Pregnancy, Brisbane, Australia; NCS = National Comorbidity Survey, USA; NCS-R = National Comorbidity Survey- Replication, USA; NEMESIS/NEMESIS-2 = Netherlands Mental Health Survey and Incidence Study (study 1: 1996-1999 and study 2: 2007-2009),

N2 = NEMESIS-2 cases; Netherlands; NESARC = National Epidemiological Survey on Alcohol and Related Conditions, USA; NoRMHS = the Northern Rivers Mental Health Study, New South Wales, Australia; NPMS = the British National Psychiatric Morbidity Survey, UK. NSMHWB = National Survey of Mental Health and Well-Being, all states, Australia; $\mathrm{OAD}=$ overanxious disorder; $\mathrm{OCD}=0 \mathrm{bsessive}$ compulsive disorder; $\mathrm{PA}=\mathrm{panic}$ attacks: $\mathrm{PD}=\mathrm{panic}$ disorder; PRIME-MD = Primary Care Evaluation of Mental Disorders; PTSD = post-traumatic stress disorder; $S A=$ separation anxiety; SAD = social anxiety disorder/social phobia; SCID-I/NP = Structured Clinical Interview for DSM-IV, non-patient version; SIAS = Social Interaction Anxiety Scale; SP = specific phobias; STAI = State-Trait Anxiety Inventory; STAI-Y = STAI for Youth; STAI-Y A = STAI state anxiety subscale; STAI-Y B = STAI trait anxiety subscale; $\mathrm{T}$ = specific wave of data collection in longitudinal studies; TH2K = Teen Health 2000 Study, Houston, USA; VAHCS = Victorian Adolescent Health Cohort Study, Victoria, Australia; YASR = Young Adult Self-Report T = specific wave of data collection in longitudinal studies; TH2K = Teen Health 2000 Study, Houston, USA; VAHCS = Victorian Adolescent Health Cohort Study, Victoria, Australia; YASR = Young Adult
*Cross-sectional was chosen if the results were obtained from one data set (even if the study was longitudinal), longitudinal refers to studies that show data at different time points (waves). **Most studies did not specify the total $N$ used to compute the ORs used in the current study. 
The heterogeneity among studies was investigated using a $Q$-statistic and an $I^{2}$ index [60]. The $Q$-statistic tests the null-hypothesis that $Q=0$ meaning that there is homogeneity in effect sizes among studies included in the analysis. The $I^{2}$ index expresses the $Q$-statistic on a $0-100 \%$ scale $\left(I^{2}=100 \% \times(Q-d f) / Q\right)$ using $d f=k-1$ ( $k=$ number of studies) and can be interpreted as the variability in effect sizes due to real differences among studies (as opposed to differences due to chance alone). The interpretation criteria for $I^{2}$ are: 25\% (little heterogeneity), 50\% (moderate heterogeneity), and 75\% (high heterogeneity) [60].

Three separate meta-analyses were performed on the data reported in Table 3:

- anxiety/no anxiety vs. cannabis use/no use,

- anxiety/no anxiety vs. CUD/no CUD (or no use),

- anxiety + depression/no diagnosis vs. cannabis use/ no use.

\section{Sensitivity and moderator analyses}

The stability of the overall mean weighted $O R$ in each analysis was investigated using one-study removed and cumulative analyses. These analyses show how the overall mean weighted $O R$ changes if one study at a time is removed from all other studies or studies are added cumulatively over time. The moderator analyses (univariate meta-regression and subgroup analysis) were used to investigate the direction of the relationship between anxiety and cannabis use and the influence of systematic differences among studies on the overall mean weighted $O R$.

\section{Publication bias analyses}

Publication bias refers to an overestimation of the overall mean weighted effect size in meta-analysis due to inclusion of studies based on large sample sizes and/or large effect sizes [57]. Such studies are more likely to be published and thus are easier to locate during a systematic literature search. In contrast, studies with smaller samples and/or small (often not statistically significant) effect sizes are either not published at all or published in smaller (often non-English language) journals that are not listed on major databases [57].

Publication bias in the current study was assessed using methods available in CMA, which are described in detail in the Additional file 1. The theoretical number of null-studies (with $O R=1$ ) required to remove the statistical significance of the overall mean weighted $O R$ was computed using Rosenthal's Fail-Safe $N$ [61]. Furthermore, the symmetry in a funnel plot of $O R$ (on a logarithmic scale) vs. SEM [62] was assessed using the Duval and Tweedie's Trim-and-Fill analysis [63]. Finally, the Begg and Mazumdar Rank Order Correlation (Kendall's tau b) [64] and Egger's regression [65] were used to investigate the relationship between the standardised $O R$ and $S E M$ or $1 / S E M$ in each study, respectively. According to these methods, a systematic publication bias might be present in meta-analysis if the Fail-Safe $N$ is small and smaller studies differ systematically (significantly) from the larger studies (funnel plot asymmetrical, correlation statistically significant, and regression intercept significantly different from zero) [57].

\section{Results}

According to three separate meta-analyses summarised in Table 4, there was a small positive association between

- $\quad$ anxiety and cannabis use $(O R=1.24,95 \% C I$ : 1.06-1.45; Figure 2),

- anxiety and cannabis use disorders (CUD; $O R=1.68$, 95\% CI: 1.23-2.31; Figure 3), and

- anxiety + depression and cannabis use $(O R=1.68$, 95\% CI: 1.17-2.40; Figure 4).

Thus, those with various anxiety disorders and concurrent anxiety + depression were more likely to use cannabis or had a CUD (dependence and/or abuse/harmful use) compared to those without anxiety disorders.

There was little evidence for a systematic publication bias because Fail-Safe $N s$ were high $(N=17$ to 105$)$, between 3 to 8 unpublished studies were needed for every study in the analysis to reduce the overall mean weighted $O R$ to 1 (Table 4), and the funnel plots were symmetrical around the overall mean weighted $O R$ (Figures 2, 3 and 4).

One-study removed analysis showed that the results of two analyses (anxiety vs. cannabis use and anxiety vs. CUD) were stable and not dependent on any one study alone (Table 4). Specifically, no study was able to eliminate the significant associations when removed from the analysis one at a time (Additional file 1: Figure S1). However, the results of the anxiety + depression vs. cannabis use analysis were dependent on one study [36]. Without this study there was only a non-significant trend $(p=.058)$ towards a positive association between anxiety + depression and cannabis use (Table 4; Additional file 1: Figure S1).

The cumulative analysis showed that the significant and positive association between anxiety and cannabis use emerged as studies published in 1998-2013 were added to the overall analysis one at a time (Table 4). The results indicate that the overall effect size was always small as new studies were added to all previous studies (Additional file 1: Figure S2). Similar conclusion can be drawn regarding the small, positive, and significant association between anxiety and 
Table 3 Odds ratios (OR) for anxiety (or anxiety + depression) vs. no disorder in cannabis users vs. non-users (or in cannabis users with CUD vs. no CUD) in

\section{$\mathbf{N}=\mathbf{3 1}$ studies}

\begin{tabular}{ll}
\hline $\begin{array}{l}\text { Study/Name (Part 1: } \\
\text { anxiety diagnoses) }\end{array}$ & Cannabis use vs. no use \\
\hline $\begin{array}{l}\text { Agosti et al., 2002; NCS, USA } \\
\text { [26] }\end{array}$ & \\
Beard et al., 2006; NoRMHS, & \\
Australia [27] & \\
Brook et al., 1998; Upstate NY, & T4 AD to T3 use vs. no use \\
USA [28] & \\
Brook et al., 2001; Columbia & T1 AD to T2 regular vs. non- \\
[29] ${ }^{2}$ & regular use \\
& T1 regular vs. non-regular use to \\
& T2 AD \\
& Combined: AD or regular use: \\
& T1 vs. T2
\end{tabular}

CUD vs. no CUD (or no use)
Current $A D$ in lifetime CD who
used within past month vs. no $C D$
T0 CUD (vs. no CUD) to T1 AD

Buckner et al., 2008; Oregon,

USA [30]

Cascone et al., 2011;

Switzerland [33]

Cougle et al., 2011; NCS-R,

USA [37 $]^{3}$

Crum et al., 1993; ECA, USA [38]

Degenhardt et al., 2001; NSMHWB, Australia [39]

Degenhardt et al., 2013;

VAHCS, Australia [41]

Fergusson et al., 1996; CHDS, New Zealand [42]

Low et al., 2008; USA [45]

Martins \& Gorelick, 2011

NESARC, USA [46]
T1 SAD to T4 CD vs. no CD

Past 12 months CD (vs. no CD) predicted with STAI-Y B

Lifetime: PTSD to use vs. no use

Past 12 months: OCD to use vs. no use

Past 12 months: $A D$ to use vs. no use

AD at 29 to $\geq$ weekly vs. no use at $15-29$

$A D$ at 16 to use vs. no use at 15 use

Past

$A D$ at 29 to $C D$ at 29 vs. no $C D$

$1.2(.5-2.8)$

Past 6 months: CA (vs. no CA) to AD

Lifetime: CUD (vs. no CUD) to AD

OR $(95 \% C l)$
Anxiety/no
anxiety in CUD/
no CUD

$2.6(1.5-4.5)$

$.78(.18-3.30)$

\begin{tabular}{|c|c|}
\hline $\begin{array}{l}\text { Data location } \\
\text { in study }\end{array}$ & $O R$ adjusted for \\
\hline Text p. 646 & No information \\
\hline Table three & $\begin{array}{l}\text { Unadjusted (comorbidity with } \\
\text { other diagnoses possible) }\end{array}$ \\
\hline Table two & Demographics, prior AD \\
\hline Table one & Demographics, cannabis use at $\mathrm{T} 1$ \\
\hline Table two & Demographics, $\mathrm{AD}$ at $\mathrm{T} 1$ \\
\hline
\end{tabular}

Demographics, AD at $\mathrm{TI}$
$4.88(1.43-16.64) \quad$ Text p. 235

1.02 (.97-1.08) Table five

D

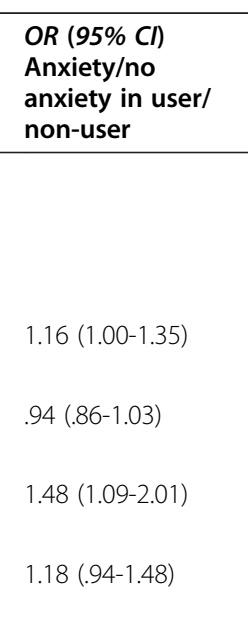

non-user

$1.16(1.00-1.35)$

$.94(.86-1.03)$

$1.48(1.09-2.01)$

$1.18(.94-1.48)$

$2.45(1.70-3.52)$

$1.54(.78-3.04)$

$.88(.60-1.29)$

$3.2(1.1-9.2)$

(1)
problems, recruitment context
Demographics, lifetime alcohol/ nicotine dependence/abuse

Excluded past or baseline OCD cases

1.40 (.84-2.37) Table four Demographics, other substance use, neuroticism

$2.2(1.1-4.4) \quad$ Table one, three

Demographics, alcohol/substance dolescent anxiety/ depression

Table three Demographics, substance use and dependence, anxiety/

depression, other mental health problems at 15

$\begin{array}{lll}1.4(.4-4.7) & \text { Table four } & \begin{array}{l}\text { Demographics, sampling site, } \\ \text { depression }\end{array} \\ 3.2(2.0-5.2) & \text { Table four } & \text { Demographics }\end{array}$


Table 3 Odds ratios (OR) for anxiety (or anxiety + depression) vs. no disorder in cannabis users vs. non-users (or in cannabis users with CUD vs. no CUD) in $\mathbf{N}=\mathbf{3 1}$ studies (Continued)

\begin{tabular}{|c|c|c|c|c|c|c|c|}
\hline \multicolumn{3}{|l|}{$\begin{array}{l}\text { Roberts et al., 2007; TH2K, } \\
\text { USA [49] }\end{array}$} & \multicolumn{2}{|l|}{$\begin{array}{l}\text { Past } 12 \text { months: AD to CUD vs. no } \\
\text { CUD }\end{array}$} & $.9(.4-2.1)$ & Table six & $\begin{array}{l}\text { Concurrent (past } 12 \text { months) } \\
\text { mood, conduct, ADHD disorders; } \\
\text { alcohol, other substance abuse/ } \\
\text { dependence }\end{array}$ \\
\hline \multicolumn{3}{|l|}{$\begin{array}{l}\text { van der Pol et al., 2013; } \\
\text { CanDep + NEMESIS-2, } \\
\text { Netherlands [51] }\end{array}$} & \multicolumn{2}{|l|}{$\begin{array}{l}\text { Past } 12 \text { months: } A D \text { to } C D \text { (vs. } \\
\text { no/non-frequent use) ( } D+\text { vs. } N 2 \\
\text { groups) }\end{array}$} & $1.12(.48-2.63)$ & Table two; Authors & $\begin{array}{l}\text { Demographics, childhood } \\
\text { adversity, tobacco (past month), } \\
\text { frequent alcohol, cocaine, ecstasy } \\
\text { use (past } 12 \text { months) }\end{array}$ \\
\hline $\begin{array}{l}\text { van Laar et al., 2007; NEMESIS, } \\
\text { Netherlands [52] }\end{array}$ & \multicolumn{2}{|c|}{ AD (T0-T2) to T0 use vs. no use } & & $1.18(.71-1.97)$ & & Table four & $\begin{array}{l}\text { Demographics, neuroticism, } \\
\text { childhood trauma, lifetime: } \\
\text { alcohol, other SUDs, psychotic } \\
\text { symptoms, AD }\end{array}$ \\
\hline $\begin{array}{l}\text { Wittchen et al., 2007; EDSP, } \\
\text { Germany [53] }\end{array}$ & \multicolumn{2}{|c|}{ Lifetime use vs. no use to T0 AD } & Lifetime CUD vs. no CUD to T0 AD & $1.5(1.1-2.1)$ & $1.7(1.1-2.5)$ & Table four & Gender \\
\hline $\begin{array}{l}\text { Zvolensky et al., 2006; CSHS, } \\
\text { USA [54] }\end{array}$ & \multicolumn{2}{|c|}{ Lifetime: use vs. no use to PA } & Lifetime: $C D$ vs. no $C D$ to $P A$ & $.89(.63-1.30)$ & $2.1(1.1-4.3)$ & Text p. 482 & $\begin{array}{l}\text { Demographics, other substance } \\
\text { use }\end{array}$ \\
\hline $\begin{array}{l}\text { Zvolensky et al., 2010; NCS-R, } \\
\text { USA [55] }\end{array}$ & \multicolumn{2}{|c|}{ Lifetime: PD to use vs. no use } & & $1.70(1.33-2.17)$ & & Table one & $\begin{array}{l}\text { Demographics, lifetime alcohol, } \\
\text { nicotine, illicit substance abuse/ } \\
\text { dependence }\end{array}$ \\
\hline NCS-R combined ${ }^{3}$ & \multicolumn{2}{|c|}{$\begin{array}{l}\text { Lifetime: PTSD + PD to use vs. no } \\
\text { use }\end{array}$} & & $2.04(1.50-2.78)$ & & & \\
\hline $\begin{array}{l}\text { Study/Name (Part 2: anxiety } \\
\text { severity scores)* }\end{array}$ & $\begin{array}{l}\text { Non-user } M \\
(S D) ; N \\
\text { (timeframe) }\end{array}$ & $\begin{array}{l}\text { Cannabis User } M \\
\text { (SD); } N \\
\text { (timeframe) }\end{array}$ & CUD $M(S D) ; N$ (timeframe) & $\begin{array}{l}\text { OR }(95 \% \mathrm{Cl}) \\
\text { Anxiety/no } \\
\text { anxiety in user/ } \\
\text { non-user }\end{array}$ & $\begin{array}{l}\text { OR }(95 \% C l) \\
\text { Anxiety/no } \\
\text { anxiety in CUD/ } \\
\text { no CUD }\end{array}$ & Location in study & $\begin{array}{l}\text { Scale (study exclusionary } \\
\text { criteria) }\end{array}$ \\
\hline $\begin{array}{l}\text { Buckner \& Schmidt, 2008; } \\
\text { USA [31] }\end{array}$ & $\begin{array}{l}23.6 \text { (11.6); } \\
105 \text { (lifetime) }\end{array}$ & $\begin{array}{l}22.2(13.1) ; 109 \\
\text { ( } \geq \text { weekly frequent } \\
\text { use; lifetime) }\end{array}$ & & $.81(.50-1.33)$ & & Table one & SIAS (none) \\
\hline $\begin{array}{l}\text { Buckner et al., 2012; } \\
\text { USA [32] }\end{array}$ & $\begin{array}{l}21.3(15.5) ; \\
66 \text { (lifetime) }\end{array}$ & $\begin{array}{l}21.7(13.7) ; 134 \\
\text { (past month) }\end{array}$ & & $1.05(.62-1.80)$ & & Table one; Authors & $\begin{array}{l}\text { SIAS (high-risk suicidal behaviour, } \\
\text { psychotic) }\end{array}$ \\
\hline $\begin{array}{l}\text { Chabrol et al., 2005; } \\
\text { France }[34]^{5}\end{array}$ & $\begin{array}{l}35.3(11.1) \\
98 \text { (past } 6 \\
\text { months) }\end{array}$ & $\begin{array}{l}37.1(10.3) ; 114 \\
\text { (past } 6 \text { months) }\end{array}$ & 38.1 (9.8); 44 (past 6 months) & $1.36(.83-2.22)$ & $1.61(.84-3.07)$ & $\begin{array}{l}\text { Table two Table } \\
\text { three }\end{array}$ & STAI A (none) \\
\hline $\begin{array}{l}\text { Chabrol et al., 2008; } \\
\text { France [35] }\end{array}$ & $\begin{array}{l}38.3(12.4) ; \\
189 \text { (past } 6 \\
\text { months) }\end{array}$ & $\begin{array}{l}42.9(13.3) ; 59 \\
\text { (past } 6 \text { months) }\end{array}$ & & $1.94(1.14-3.30)$ & & Table one & STAI A (none) \\
\hline $\begin{array}{l}\text { Lamers et al., 2006; } \\
\text { USA [44] }\end{array}$ & $\begin{array}{l}4.0(4.3) ; 15 \\
\text { (past } 12 \\
\text { months) }\end{array}$ & $\begin{array}{l}2.9(1.9) ; 15 \\
\text { (lifetime) }\end{array}$ & & $.55(.15-2.03)$ & & Table three & $\begin{array}{l}\text { BAI (alcohol, drug dependence, } \\
\text { schizophrenia, depression, } \\
\text { antisocial behaviour, } \\
\text { psychoactive drug use) }\end{array}$ \\
\hline
\end{tabular}


Table 3 Odds ratios (OR) for anxiety (or anxiety + depression) vs. no disorder in cannabis users vs. non-users (or in cannabis users with CUD vs. no CUD) in $\mathbf{N}=\mathbf{3 1}$ studies (Continued)

\begin{tabular}{|c|c|c|c|c|c|c|}
\hline $\begin{array}{l}\text { Study/Name (Part 3: } \\
\text { anxiety + depression, AMD) }\end{array}$ & Cannabis use vs. no use & CUD vs. no CUD (or no use) & $\begin{array}{l}\text { OR }(95 \% \mathrm{Cl}) \mathrm{AMD} / \\
\text { no AMD in user/ } \\
\text { non-user }\end{array}$ & $\begin{array}{l}\text { OR }(95 \% \mathrm{Cl}) \\
\text { AMD/no AMD in } \\
\text { CUD/no CUD }\end{array}$ & Location in study & $O R$ adjusted for \\
\hline $\begin{array}{l}\text { Cheung et al., 2010; CAMH, } \\
\text { Canada [36] }\end{array}$ & $\begin{array}{l}\text { Past } 12 \text { months: AMD to daily } \\
\text { use vs. no use }\end{array}$ & & $2.05(1.18-2.93)$ & & Table two & Demographics, alcohol misuse \\
\hline $\begin{array}{l}\text { Degenhardt et al., 2010; } \\
\text { VAHCS, Australia [40] }]^{6}\end{array}$ & $\begin{array}{l}\text { AMD at } 24 \text { (wave } 8 \text { ) to weekly }+ \\
\text { use vs. no use past } 6 \text { months at } \\
15-17 \text { (wave 1-6) }\end{array}$ & & $.88(.55-1.40)$ & & Table two & $\begin{array}{l}\text { Demographics, adolescent: AMD, } \\
\text { alcohol, nicotine use }\end{array}$ \\
\hline $\begin{array}{l}\text { Hayatbakhsh et al., 2007; } \\
\text { MUSP, Australia [43] }\end{array}$ & $\begin{array}{l}\text { AMD at } 21 \text { to frequent (past } \\
\text { month) vs. never used drugs } \\
\text { (lifetime) }\end{array}$ & & $2.1(1.1-4.0)$ & & Table four & $\begin{array}{l}\text { Demographics, no other illicit } \\
\text { drugs, maternal and adolescent: } \\
\text { AMD, alcohol, nicotine use }\end{array}$ \\
\hline $\begin{array}{l}\text { McGee et al., 2000; DMHDS, } \\
\text { New Zealand [47] }\end{array}$ & $\begin{array}{l}\text { Internalising disorders at } 15 \text { to } \\
\text { use vs. no use past } 12 \text { months at } \\
15\end{array}$ & & $2.45(1.41-4.25)$ & & Table five & $\begin{array}{l}\text { Unadjusted (adjusted OR could } \\
\text { not be used because } 95 \% \mathrm{Cl} \\
\text { were not reported) }\end{array}$ \\
\hline $\begin{array}{l}\text { NPMS, UK; appendix, Moore } \\
\text { et al., } 2007 \text { [10] }\end{array}$ & $\begin{array}{l}\text { AMD (CIS-R } \geq 12) \text { to ever use vs. } \\
\text { no use }\end{array}$ & AMD (CIS-R $\geq 12)$ to $C D$ vs. no $C D$ & $.8(.4-1.6)$ & $.9(.2-3.6)$ & p. IV & $\begin{array}{l}\text { Excluded if baseline } C I S-R \geq 12 \text {, } \\
\text { demographics, other drugs, } \\
\text { alcohol, nicotine use }\end{array}$ \\
\hline $\begin{array}{l}\text { Patton et al., 2002; VAHCS, } \\
\text { Australia [48] }]^{6}\end{array}$ & $\begin{array}{l}\text { AMD (CIS-R } \geq 12 \text { ) at } 21 \text { (wave } 7 \text { ) } \\
\text { to }<\text { weekly use vs. no use past } 6 \\
\text { months at } 15-17 \text { (wave } 1-6 \text { ) }\end{array}$ & & $1.4(.94-2.0)$ & & Table three & $\begin{array}{l}\text { AMD at 15-17, alcohol use, } \\
\text { parental demographics }\end{array}$ \\
\hline $\begin{array}{l}\text { Swift et al., 2008; VAHCS, } \\
\text { Australia }[50]^{6}\end{array}$ & $\begin{array}{l}\text { Weekly }+ \text { use vs. no use (past } 12 \\
\text { months at } 24 \text {, wave } 8 \text {, who used } \\
\text { cannabis at } 15-17 \text {, waves } 1-6 \text { ) to } \\
\text { AMD (CIS-R }>11 \text { ) at } 15-17 \text { (at } 3 / 6 \\
\text { waves of wave } 1-6 \text { ) }\end{array}$ & $\begin{array}{l}\text { CD vs. no CD (past } 12 \text { months at } \\
24 \text {, wave } 8 \text {, who used cannabis } \\
\text { at } 15-17 \text {, waves } 1-6 \text { ) to AMD } \\
\text { (CIS-R> 11) at } 15-17 \text { (at } 3 / 6 \\
\text { waves of wave 1-6) }\end{array}$ & $2.0(1.0-3.8)$ & $1.4(.71-2.9)$ & Table four & $\begin{array}{l}\text { Demographics, adolescent: } \\
\text { maximum level of cannabis use, } \\
\text { nicotine and alcohol use, } \\
\text { antisocial behaviour }\end{array}$ \\
\hline
\end{tabular}

VAHCS combined ${ }^{6}$

AMD at $15-24$ to at least <

Notes: For abbreviations refer to Table 2 .

'The $O R$ was computed based on the following $N$ of cases in the 'Anxiety' column and 'Baseline No Diagnosis' and 'Cannabis Diagnosis' rows reported in Table three of the article: $N=2$ (CUD/anxiety), $N=46$ (CUD/no anxiety), $N=51$ (no CUD/anxiety), $N=914$ (no CUD/no anxiety).

${ }^{2}$ The two ORs were combined according to the formulae for combining dependent effect sizes shown in the Additional file 1.

${ }^{3}$ Both studies reported ORs based on the same number of cases from the same study (NCS-R). It was assumed that both studies were dependent (same cases might have been used to compute the ORs in both studies). Thus, both $O R$ s were combined into one common $O R$ that was used in all subsequent analyses using the formulae shown in the Additional file 1.

${ }^{4}$ The $O R$ was computed based on the following $N$ of cases in the 'Cases' (OCD) vs. 'Non-cases' columns and 'Use of marijuana only' and 'No drug use' rows reported in Table one of the article: $N=12$ (use/anxiety),

$N=42$ (use/no anxiety), $N=82$ (no use/anxiety), $N=441$ (no use/no anxiety). The risk ratio (RR), adjusted for confounders, was also reported in the study (Table two). However, $R R$ and $O R$ are not equivalent [56] and thus unadjusted $O R$ is computed here which is more conservative than the $R R$ in Table two of the study $(R R=2.1,95 \% \mathrm{Cl}: 1.0-4.5$ ).

${ }^{5}$ The STAI A scores reported separately for girls and boys were combined into one score in each of the three groups- non-users, users, and users with CUD using the formulae shown in the Additional file 1. The ORs in this study were computed based on these combined scores since all other studies in the current analysis reported anxiety scores in both genders combined rather than separately.

${ }^{6}$ The $O R s$ in studies utilising VAHCS data from waves $1-8$ were combined according to the formulae for combining dependent effect sizes shown in the Additional file 1 .

${ }^{7}$ The $O R$ was computed based on the following $N$ of cases in the 'Cannabis use at age $15^{\prime}$ and 'Mental disorder- Internal (anxiety and depression)' columns reported in Table five of the article: $N=20$ (use/internal), $N=62$ (use/no disorder), $N=84$ (no use/internal), $N=637$ (no use/no disorder).

*The standardised mean difference (Cohen's $d$ ) was computed for user - non-user or CUD - non-user groups in all studies in Part 2 of this table. This effect size was then converted into OR using the formulae shown in the Additional file 1 . 


\begin{tabular}{|c|c|c|c|c|}
\hline & & $\begin{array}{l}\text { Anxiety vs. use/no use } \\
N=15 \text { (Figure } 2 \text { ) }\end{array}$ & $\begin{array}{l}\text { Anxiety vs. CUD/no CUD } \\
\text { (or no use) } N=13 \text { (Figure } 3 \text { ) }\end{array}$ & $\begin{array}{l}\text { Anxiety + depression vs. use/ } \\
\text { no use } N=5 \text { (Figure } 4 \text { ) }\end{array}$ \\
\hline Overall mean weighted $O R$ & OR (95\% Cl) p ptwo-tailed & $1.24(1.06-1.45) .006^{*}$ & $1.68(1.23-2.31) .001^{*}$ & $1.68(1.17-2.40) .004^{*}$ \\
\hline Heterogeneity statistics & $\begin{array}{l}Q(d f) \\
p_{P^{\text {two-tailed }}}\end{array}$ & $\begin{array}{l}30(14) \\
.009^{*} \\
53 \%\end{array}$ & $\begin{array}{l}55(12) \\
<.0001^{*} \\
78 \%\end{array}$ & $\begin{array}{l}8(4) \\
.091 \\
50 \%\end{array}$ \\
\hline $\begin{array}{l}\text { One-study removed analysis } \\
\text { (Additional file 1: Figure S1) }\end{array}$ & $\begin{array}{l}\text { Which studies, removed one at a time from the analysis, } \\
\text { remove the significance of the overall mean weighted OR? }\end{array}$ & None & None & Cheung et al., 2010 [36] \\
\hline $\begin{array}{l}\text { Cumulative analysis (1993 to 2013) } \\
\text { (Additional file 1: Figure S2) }\end{array}$ & $\begin{array}{l}\text { Which studies, added to all previous studies one at a time, } \\
\text { remove the significance of the overall mean weighted OR? }\end{array}$ & $\begin{array}{l}\text { Crum et al., } 1993 \text { [38]; } \\
\text { Fergusson et al., } 1996[42]\end{array}$ & Degenhardt et al., 2001 [39] & NPMS, 2007 [10] \\
\hline Rosenthal's Fail-safe $N$ for $p>.05$ & $\begin{array}{l}N \text { studies needed to remove the significance of the overall } \\
\text { mean weighted } O R \text { ( } N \text { studies missing for every study in } \\
\text { meta-analysis needed to remove the significance of the } \\
\text { overall mean weighted } O R \text { ) }\end{array}$ & $\begin{array}{l}N=49 \\
(49 / 15=3)\end{array}$ & $\begin{array}{l}N=105 \\
(105 / 13=8)\end{array}$ & $\begin{array}{l}N=17 \\
(17 / 5=3)\end{array}$ \\
\hline \multirow{2}{*}{$\begin{array}{l}\text { Duval and Tweedie's Trim-and-Fill } \\
\text { analysis }\end{array}$} & \multirow{2}{*}{$\begin{array}{l}\text { Funnel plot symmetrical? } N \text { studies missing on either side } \\
\text { of the overall mean weighted } O R\end{array}$} & YES & YES & YES \\
\hline & & $N=0$ & $N=0$ & $N=0$ \\
\hline \multirow{2}{*}{$\begin{array}{l}\text { Begg and Mazumdar Rank Order } \\
\text { Correlation }\end{array}$} & $\tau$ & .13 & -.19 & -.30 \\
\hline & $p_{\text {two-tailed }}$ & .488 & .360 & .462 \\
\hline \multirow[t]{2}{*}{ Egger's regression } & intercept & .25 & 1.73 & -4.05 \\
\hline & $p_{\text {two-tailed }}$ & .748 & $.005^{*}$ & .415 \\
\hline
\end{tabular}

Note: Figure numbers refer to forest plots and funnel plots for each analysis (Figures S1 and S2 can be found in the Additional file 1).

Abbreviations: CUD cannabis use disorder (cannabis dependence and/or abuse/harmful use), NPMS the British National Psychiatric Morbidity Survey, UK; $\tau=$ Kendall's correlation coefficient tau $b$ with continuity correction. ${ }^{*} p<.05$. 


Study name
Crum et al. 1993 [38]
Fergusson et al. 1996 [42]
Brook et al. 1998 [28]
Brook et al. 2001 [29] combined
Degenhardt et al. 2001 [39]
Chabrol et al. 2005 [34]
Lamers et al. 2006 [44]
Zvolensky et al. 2006 [54]
van Laar et al. 2007 [52]
Wittchen et al. 2007 [53]
Buckner \& Schmidt 2008 [31]
Chabrol et al. 2008 [35]
NCS-R 2010 [37,55] combined
Buckner et al. 2012 [32]
Degenhardt et al. 2013 [41]

\begin{tabular}{cccc}
\multicolumn{5}{c}{ Statistics for each study } \\
$\begin{array}{c}\text { Odds } \\
\text { ratio }\end{array}$ & $\begin{array}{c}\text { Lower } \\
\text { limit }\end{array}$ & $\begin{array}{c}\text { Upper } \\
\text { limit }\end{array}$ & p-Value \\
1.54 & 0.78 & 3.04 & 0.218 \\
1.20 & 0.51 & 2.84 & 0.678 \\
1.16 & 1.00 & 1.35 & 0.053 \\
1.18 & 0.94 & 1.48 & 0.153 \\
0.88 & 0.60 & 1.29 & 0.513 \\
1.36 & 0.83 & 2.22 & 0.222 \\
0.55 & 0.15 & 2.03 & 0.368 \\
0.89 & 0.62 & 1.28 & 0.528 \\
1.18 & 0.71 & 1.97 & 0.525 \\
1.50 & 1.09 & 2.07 & 0.014 \\
0.81 & 0.50 & 1.33 & 0.409 \\
1.94 & 1.14 & 3.30 & 0.015 \\
2.04 & 1.50 & 2.78 & 0.000 \\
1.05 & 0.62 & 1.80 & 0.853 \\
3.20 & 1.11 & 9.25 & 0.032 \\
1.24 & 1.06 & 1.45 & 0.006
\end{tabular}

\section{Odds ratio and $95 \% \mathrm{Cl}$}

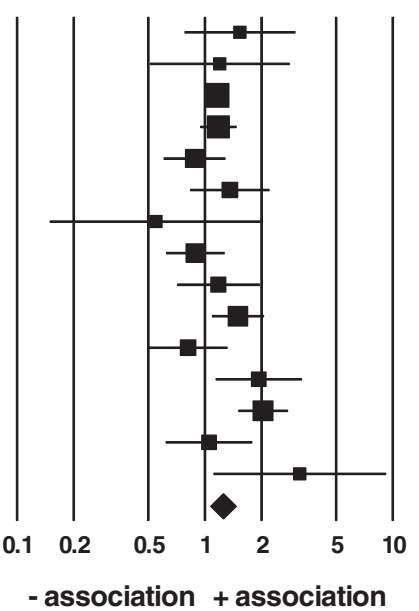

Funnel Plot of Standard Error by Log odds ratio

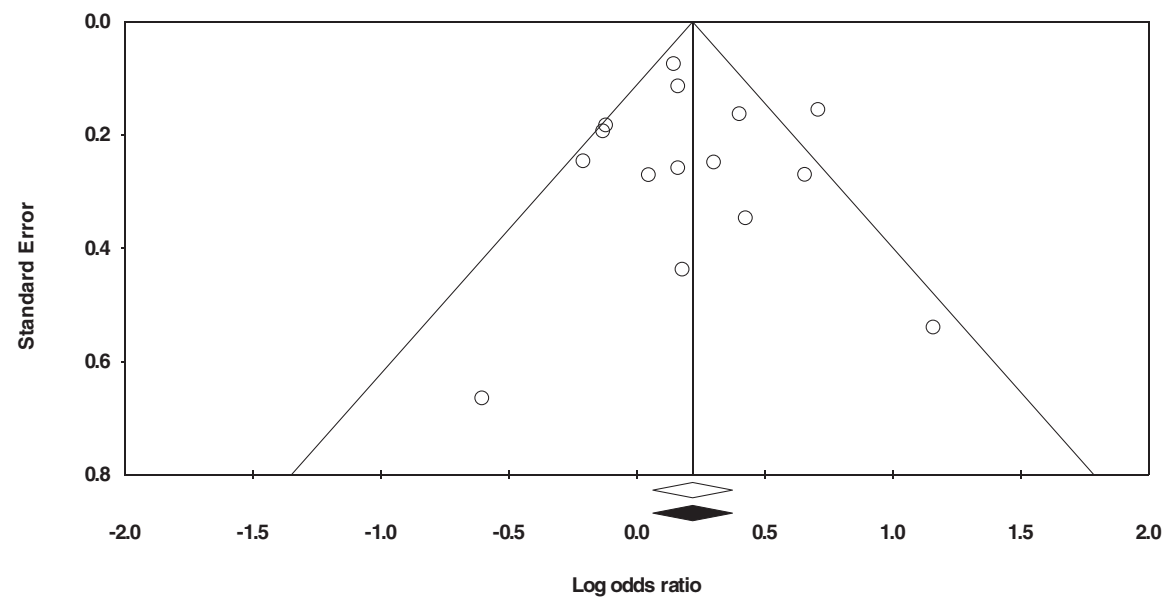

Figure 2 Random-effects meta-analysis of $\boldsymbol{N}=15$ studies on anxiety vs. cannabis use/no use. Notes: The forest plot (top) shows the effect size $(O R)$ in each study, the weight of each study (size of the box), and the $95 \% \mathrm{Cl}$ (the horizontal line through each box). The overall mean weighted $O R$ is depicted as the centre of the diamond and its horizontal edges are the $95 \% \mathrm{Cl}$. Since the diamond did not cross the line of no effect $(O R=1)$, there was an overall positive association between anxiety and cannabis use in $N=15$ studies (overall mean weighted $O R=1.24$, 95\% Cl: 1.06-1.45). The funnel plot (bottom) shows the distribution of the individual effect sizes around the overall mean weighted OR (unfilled diamond). The trim-and-fill analysis revealed that the plot was symmetrical (the recomputed overall mean weighted OR depicted as the filled diamond overlaps with the unfilled diamond) suggesting that there was little evidence for a publication bias in the current analysis.

CUD that emerged in studies published in 2002-2013 and remained consistently small. In contrast, adding the results of the NPMS study [10] either eliminated or reduced the positive association between anxiety + depression vs. cannabis use in studies published in 2000-2010 (Additional file 1: Figure S2).

As expected there was a moderate-high heterogeneity among the effect sizes in the three meta-analyses (Table 4). Specifically, according to the $I^{2}$ index, between $50-78 \%$ of variability in ORs was due to real differences among studies rather than chance. The inspection of study characteristics in Tables 2 and 3 revealed that such heterogeneity was probably due to two hypothesised systematic differences among studies:

(1) OR adjustment- in some studies ORs were controlled for confounders, such as other substance use, other substance use disorders, past anxiety, other psychiatric illness, and demographics (age, gender) while in other studies no statistical adjustment of ORs was used,

(2) year of publication- more recent studies might have applied more complex statistical modelling techniques to adjust ORs compared to the older studies regardless of when the data were actually collected. 
One additional systematic difference among studies emerged only after the data were extracted from all studies (Tables 2 and 3):

(3) diagnosis of anxiety- in some studies cases had clinical diagnoses (DSM/ICD-based) while in other studies only the severity of anxiety scores on standardised instruments were reported (these studies were classified as having 'non-clinical diagnoses' for the purposes of the current analysis).

Taking into account these differences among studies, two types of moderator analyses were performed: subgroup analyses to compare the overall mean weighted ORs in subgroups of studies above and a univariate metaregression to investigate if the year of publication could predict weighted ORs in all studies. Moderator analyses were not performed on studies with anxiety + depression because there was only one such study with unadjusted $O R$ and one study with a clinical diagnosis of anxiety + depression.

According to the subgroup analyses, the majority of studies included in the current meta-analysis reported ORs adjusted for potential confounders (Table 5). Interestingly, the small positive association between anxiety and cannabis use (or CUD) was still present even after the adjustment for such confounders (Table 5; Additional file 1: Figure S3). Therefore, it appears that the higher 
odds for anxiety in cannabis users (with or without CUD) are not exclusively due to the effects of other substances, psychiatric illnesses, or demographics.

There were too few studies $(N=3)$ to reliably investigate these associations in studies with ORs unadjusted for confounders. There was a trend for a positive association between anxiety vs. CUD in $N=3$ studies with unadjusted ORs (Table 5; Additional file 1: Figure S3).

Furthermore, the majority of current studies included cases with clinical diagnoses of anxiety according to DSM and/or ICD diagnostic systems. The small positive association between anxiety and cannabis use (or CUD) was present in the subgroups of studies with clinical diagnoses of anxiety (Table 5; Additional file 1: Figure S3). Thus, cannabis users with or without CUD have higher odds of clinically-relevant anxiety symptoms. There was a trend towards lack of such associations in the small subgroups of studies without clinical diagnoses of anxiety (Table 5; Additional file 1: Figure S3). Furthermore, according to the meta-regression, the weighted ORs were not univariately associated with the year of publication in studies included in the current analysis (Table 5).

Finally, even though a positive association does not provide evidence for causation, it was possible to investigate the temporal relationship between anxiety and cannabis use in a small subset of $N=5$ studies that reported ORs for cannabis use at baseline and anxiety at follow-up. This analysis showed that a cohort of those using cannabis at baseline was significantly more likely to have symptoms of anxiety at follow-up in studies adjusted for confounders $(O R=1.28$, 95\% CI: 1.06-1.54, $p=.01 ; N=5$ studies; Figure 5A). The opposite relationship was investigated in only one study [29]. The results showed that there was no association between anxiety at baseline and a regular cannabis use at follow-up $(O R=.94,95 \% C I$ : .86-1.03; Table 3). Interestingly, the one-study removed analysis (Figure $5 \mathrm{~B}$ ) showed that the positive association between cannabis use at baseline and anxiety at follow-up was not entirely due to the study by Degenhardt and colleagues [41] with the largest 
Table 5 Results of the moderator analyses (subgroup-analyses and univariate meta-regression)

\begin{tabular}{|c|c|c|c|c|c|c|c|}
\hline & \multirow{2}{*}{$\begin{array}{l}\text { Subgroup } \\
\text { of studies }\end{array}$} & \multicolumn{3}{|c|}{ Anxiety vs. cannabis use/no use } & \multicolumn{3}{|c|}{ Anxiety vs. CUD/no CUD (or no use) } \\
\hline & & $N$ studies & OR $(95 \% \mathrm{Cl})$ & $p_{\text {two-tailed }}$ & $N$ studies & OR $(95 \% \mathrm{Cl})$ & $p_{\text {two-tailed }}$ \\
\hline Overall mean weighted $O R$ & & 15 & $1.24(1.06-1.45)$ & $.006^{*}$ & 13 & $1.68(1.23-2.31)$ & $.001^{*}$ \\
\hline \multicolumn{8}{|l|}{ Subgroup analyses (Figure S3) } \\
\hline \multirow[t]{3}{*}{ ORs adjusted for confounders } & Yes & 12 & $1.24(1.04-1.47)$ & $.014^{*}$ & 10 & $1.66(1.17-2.37)$ & $.005^{*}$ \\
\hline & No & 3 & $1.28(.78-2.08)$ & .326 & 3 & $1.86(1.10-3.15)$ & $.021^{*}$ \\
\hline & Yes vs. No & \multicolumn{3}{|c|}{$Q(1)=.04 ; p=.848$} & \multicolumn{3}{|c|}{$Q(1)=8.96 ; p=.003^{*}$} \\
\hline \multirow{3}{*}{$\begin{array}{l}\text { Clinical diagnosis of anxiety } \\
\text { (based on DSM/ICD) }\end{array}$} & Yes & 9 & $1.29(1.04-1.61)$ & $.021^{*}$ & \multicolumn{3}{|c|}{ स } \\
\hline & No & 6 & $1.17(.93-1.48)$ & .186 & 2 & $1.14(.78-1.66)$ & .509 \\
\hline & Yes vs. No & \multicolumn{3}{|c|}{$Q(1)=.30 ; p=.586$} & \multicolumn{3}{|c|}{$Q(1)=36.91 ; p<.001^{*}$} \\
\hline Meta-regression & & $N$ studies & slope & slope $p_{\text {two-tailed }}$ & $N$ studies & slope & slope $p_{\text {two-tailed }}$ \\
\hline \multicolumn{8}{|l|}{$\begin{array}{l}\text { Predictor: Year of publication } \\
\text { Outcome: weighted } O R\end{array}$} \\
\hline \multicolumn{8}{|c|}{$\begin{array}{l}\text { Note: Confounders were: other substance use and/or other substance use disorders and/or past AD and/or other psychiatric illnesses and/or demographics. The } \\
\text { subgroup analyses were conducted using the so-called mixed-effects model of meta-analysis [57]. According to this model the studies within each subgroup were } \\
\text { combined using the random-effects model. However, since the number of subgroups was fixed (rather than randomly selected out of many subgroups), the } \\
\text { overall mean weighted ORs in both groups were compared statistically using the between-groups } Q \text {-statistic based on the fixed-effect model of meta-analysis } \\
\text { with } d f=1 \text { (number of subgroups-1). This approach to comparing ORs in independent subgroups of studies is equivalent to the independent samples } t \text {-test. } \\
\text { Figure S3 is located in the Additional file 1. }\end{array}$} \\
\hline
\end{tabular}

effect size $(O R=3.20)$ in the analysis of $N=5$ studies. However, the exclusion of the study by Brook and colleagues [29] removed the traditional level of statistical significance from the association, most likely due the low statistical power of the analysis of $N=4$ studies only. The inspection of the effect sizes alone suggests that there was a trend towards a consistently small and positive relationship between cannabis use at baseline and anxiety at follow-up (OR range: 1.21-1.44) after the removal of each of the five studies, one at a time (Figure $5 \mathrm{~B}$ ).

\section{Discussion}

The current study is the first meta-analysis to quantitatively describe the relationship between anxiety and cannabis use using data from $N=31$ studies on samples drawn from approximately 112,000 cases from the general population of 10 countries (Australia, Canada, Columbia, France, Germany, Netherlands, New Zealand, Switzerland, UK, USA). The main finding of the current meta-analysis is that cohorts with anxiety are more likely to use cannabis (odds of 1.24) or have a cannabis use disorder (odds of 1.68; Table 4). Similarly, cohorts with concurrent anxiety and depression are also more likely to use cannabis (odds of 1.68; Table 4). These findings are based on samples from the general population neither in treatment for anxiety nor cannabis use disorders. The current study quantitatively supplements the findings of one other systematic review on the relationship between anxiety and cannabis use in $N=7$ studies [10].

It can only be speculated that the associations above would be higher in samples perceiving their anxiety and/ or cannabis use as problematic and thus seeking professional treatment for either one or both of these conditions. In support of this statement, our results show a trend towards a higher and more stable relationship between anxiety and cannabis use disorders that might eventually require treatment $(O R=1.68,95 \% C I: 1.23$ $2.31)$, than cannabis use alone $(O R=1.24,95 \% C I$ : 1.06 1.45; Table 4). Similarly, higher rates of comorbidity would be expected in vulnerable populations often not included in the population-based surveys due to being homeless, imprisoned, or inpatient in psychiatric or rehabilitation institutions [66].

All three overall effect sizes were only small and their 95\% CI were close to the line of no effect $(O R=1)$ in the current study (Table 4). Such small ORs were probably due to the heterogeneous duration and definition of 'anxiety disorders' and 'cannabis use/CUD' used in the current analysis. In general, the duration of diagnoses and/or cannabis use/CUD ranged from within past 12 months to lifetime (Table 2). Furthermore, some studies investigated a wide range of anxiety diagnoses according to DSM/ICD criteria, while others focused on 'narrow' diagnoses, such as PTSD or OCD, only. Similarly, the definition of 'cannabis use' ranged between 'use $>1 \times$ (lifetime)' to 'use > daily (last 6 months)' and the prevalence of particularly heavy cannabis use (>daily) was low (Table 2). Since the total lifetime duration of cannabis use was often not reported in studies, it was not possible to further investigate the differences in results among subgroups of studies with more homogenous definition, frequency, and/or duration of cannabis use. 


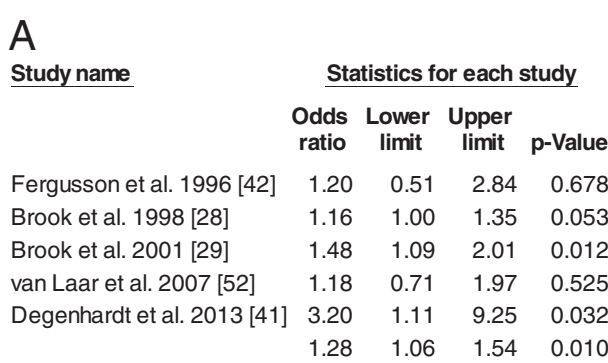

B

Study name

\begin{tabular}{lcccc}
\hline & \multicolumn{3}{c}{ Lower } & Upper \\
& Point & limit & limit & p-Value \\
Fergusson et al. 1996 [42] & 1.31 & 1.04 & 1.65 & 0.023 \\
Brook et al. 1998 [28] & 1.44 & 1.13 & 1.84 & 0.003 \\
Brook et al. 2001 [29] & 1.21 & 0.98 & 1.51 & 0.079 \\
van Laar et al. 2007 [52] & 1.33 & 1.03 & 1.72 & 0.028 \\
Degenhardt et al. 2013 [41] & 1.21 & 1.07 & 1.38 & 0.003 \\
& 1.28 & 1.06 & 1.54 & 0.010
\end{tabular}

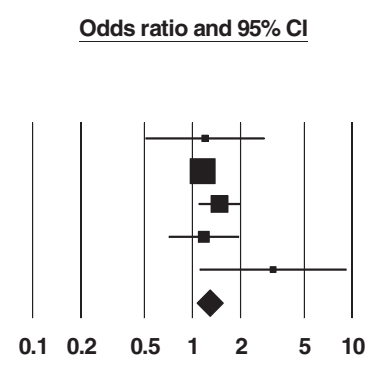

- association + association

Odds ratio $(95 \% \mathrm{Cl})$ with study removed

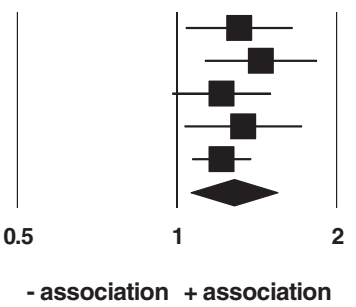

Figure 5 Random-effects meta-analysis (A) and one-study removed analysis (B) of $N=5$ studies on cannabis use at baseline and anxiety at follow-up (all ORs adjusted for potential confounders). Notes: The top forest plot (A) shows that there was an overall positive association between cannabis use at baseline and anxiety at follow-up in $N=5$ studies according to the random-effects meta-analysis (overall mean weighted $O R=1.28,95 \%$ Cl: 1.06-1.54). The bottom forest plot (B) shows the outcome of the one-study removed sensitivity analysis. 'Point' on plot $B$ refers to the overall mean weighted $O R$ of all studies without the study in each row. This analysis revealed that the positive association between cannabis use at baseline and anxiety at follow-up was still present when the study with the largest $O R$ in plot $A(O R=3.20)$ was removed from the analysis.

It is interesting that the relationship between anxiety and cannabis use was still present and positive even after controlling for confounders (other substance use/psychiatric comorbidity/demographics) in studies with mostly nonfrequent users without cannabis use disorders in the general population (Table 5). Furthermore, such positive association was found only in studies using cases with clinical symptoms of anxiety disorders (according to DSM/ICD criteria), but not in studies that measured severity of anxiety using standardised scales or symptom checklists (Table 5). It has been suggested that particularly a high-dose cannabis use in drug-naïve users could either induce some acute symptoms of anxiety (intense fear, panic attacks) without necessarily causing anxiety disorders or result from withdrawal in those with cannabis dependence [16]. Although we have not controlled for the acute cannabis use (dose, concentration of cannabinoids in urine, withdrawal symptoms), our results show that any level of cannabis use is positively related only to clinically relevant symptoms of anxiety. Thus, it is unlikely that the positive association between anxiety and cannabis use in the current study was due to exclusively acute (non-clinical) symptoms of anxiety induced by a heavy (acute) cannabis use.
In terms of direction, cannabis use could further exacerbate existing symptoms of anxiety depending on the genetic vulnerability, severity of anxiety symptoms, gender, and age, among other factors [16]. In fact, we have found that baseline cannabis use was indeed positively associated with anxiety at follow-up in $N=5$ studies that reported ORs controlled for potential confounders and four of which used the clinical diagnoses of anxiety (Figure 5). Although it is tempting to state that cannabis use at baseline caused anxiety at follow-up (Figure 5), there is little direct evidence to support such a conclusion in the current study. The effect size of this relationship had little statistical stability in terms of only a small positive value $(O R=$ 1.28 ) and the $95 \%$ confidence interval located close to the line of no effect $(O R=1)$. Furthermore, other relevant factors necessary to establish causality in epidemiological research, such as the time order, misclassification, and residual confounding $[67,68]$, were not assessed in the current analysis. Specifically, our results are based on cohorts of cases with generally low proportions of (mostly low-level) cannabis users who could have changed their group membership over time due to other factors than cannabis use alone. For example, a non-user with no 
anxiety at baseline could have become a user (due to a one-off use) with a diagnosis of anxiety related to a specific event (such as PTSD resulting from a traffic accident) at follow-up. Another plausible alternative would be that some cases were misclassified because of the slow onset of some anxiety disorders or low severity of anxiety symptoms already present at baseline but insufficient for a formal clinical diagnosis until a later point in time (at follow-up). In general, a lack of longitudinal follow-up of the same cases makes it difficult to validly and reliably study the causation in the association between any mental illness and cannabis use. Furthermore, the individual ORs were adjusted for different potential confounders in the $N=5$ primary studies (Table 3 ). This is of importance because our observed positive association might have resulted from (a) residual confounding due to inadequate controlling for potential confounders in primary studies as well as our analysis and/or from (b) multiple, unmeasured factors causally related to each other, that were not taken into account in both the primary studies and our analysis [67]. For example, we have used an unspecific and broad binary criterion to classify studies based on presence/ absence of statistical adjustment of $O R$ for any relevant confounders in our analysis (Table 5). However, it is likely that including specific confounders measured as scale variables would have been a more effective strategy to reduce their direct or indirect effect on our association. One obvious candidate for such a scale confounder could be the heaviness of cannabis use measured as frequency/day, total length of use (in years), and/or dose of cannabis/day. Other important confounders, possibly causally related to heaviness of cannabis use, could include age, severity of comorbid psychiatric diagnoses, and use of other substances. However, it must be stressed that meta-analysis utilises effect sizes based on group data from primary studies. Thus adjustments for the same (multiple) potential confounders would need to be applied in all primary studies using individual case data to be systematically carried over to a meta-analysis. The only sensitivity analysis that we were able to conduct on such a small number of studies included in our analysis $(N=5)$ was the one-study removed analysis (Figure 5B). This analysis showed that there was a trend towards a positive (but small) association between cannabis use at baseline and anxiety at follow-up even after the removal of the study with the largest effect size of $O R=3.20$ [41] from this analysis. Therefore, regardless of causation, it appears that the relationship between cannabis use at baseline and anxiety at follow-up is (at most) only small.

Due to lack of data we were unable to investigate the relationship between anxiety at baseline and cannabis use at follow-up. Presence of such a (positive) relationship could be used to support the self-medication hypothesis suggesting that those with anxiety disorders could use cannabis to relax and better cope with stress [16]. The selfmedication hypothesis would also need to be investigated taking into account the difference between the acute and long-term effects of cannabis use and the frequency/dose/ total duration of cannabis use. In general, it appears that cannabis has biphasic or bidirectional effect on anxiety [18]. Thus, those with anxiety could experience some acute relief from their symptoms after low-frequency and lowdose cannabis use. However, a regular and heavier use could lead to development of cannabis use disorders and, in turn, be associated with worsening of anxiety symptoms. These biphasic relationships could result from a dosedependent interaction between the active ingredient of cannabis (delta-9-tetrahydrocannabinol) and (dysregulation of) endocannabinoid and neurotransmitter systems, including dopamine, GABA, glutamate, serotonin, and noradrenaline $[16,18]$. Current results suggest that particularly cannabis use disorders (likely resulting from heavy use) are related to anxiety (Table 4). Similarly, dependent frequent cannabis users, but not the frequent users without dependence, had more anxiety disorders compared to the general population [51]. Furthermore, the age of initial cannabis use and/or development of cannabis use disorders and anxiety symptoms might be a necessary factor to control for when testing the association between anxiety and cannabis use. For example, early cannabis use may affect the adolescent neuromaturation and cognitive functioning [69] and thus predispose the users to subsequent development of anxiety and other mental health problems [23]. However, genetic vulnerability to anxiety could also contribute to poor early social and cognitive functioning, limited educational and employment prospects, and subsequent onset of substance use [16].

The current results could have some implications for policy making purposes. An association of a small magnitude between anxiety and cannabis use supports the argument that compared to the impact of other illicit drugs, alcohol, or tobacco, the sole role of cannabis use in public health could be judged as only modest [24] and cannabis use is only a minor contributor to the overall disease burden worldwide [8]. Thus, the current results do not provide a strong evidence for prohibiting cannabis. However, because the two conditions co-exist at a rate higher than statistical chance alone and, since cannabis use and anxiety disorders are common particularly among the younger generations, governments should focus on providing affordable, easily accessible, and effective mental health care. According to the World Mental Health (WMH) Surveys, mental disorders, including anxiety and substance abuse, are a crucial societal issue because they contribute to a loss of 'healthy life years' in terms of reduced working capacity and the required treatment [70]. Such a burden should be particularly addressed in times of increased daily stress and financial insecurity that are likely to 
contribute to development of some anxiety disorders. Major financial problems were also identified as more important predictors of development of cannabis dependence than other 'stable' vulnerability factors (such as childhood adversity, own or family history of mental illness or problematic substance use, and personality) [71].

It is difficult to effectively treat comorbidity and to quantify the recovery from such a comorbidity to a meaningful life [66]. In general, patients with dual diagnoses (problematic substance use and mental health comorbidity) are more satisfied with integrated comorbidity treatment than with standard treatment without explicit focus on both diagnoses [72]. Detecting and addressing cannabis use in patients presenting with anxiety disorders might also be important because cannabinoids could theoretically interact with any pharmacological treatment for anxiety. However, the accuracy of self-reported cannabis use depends on appropriate conditions of data collection, including confidentiality and anonymity [73]. Such conditions could in turn improve the accuracy of psychiatric diagnosis, and contribute to more patients receiving treatment for substance use disorders in the clinical practice [74]. Since particularly the infrequent users rarely seek professional help, it might be useful to include anxiety as one risk associated with cannabis use in educational programs on the level of schools, community health centres, and in the context of primary care settings $[10,40]$. Especially the motivational interviewing and motivational enhancement techniques might be useful for approaching cannabis use disorders because young people often lack the motivation to address their substance use and thus miss early intervention strategies [33,50]. Reducing social stigma and providing informal care could also contribute to more problematic cannabis users (with cannabis use disorders) subsequently seeking professional treatment [75]. Furthermore, recovery from comorbidity might be enhanced by using social networks and peer education and support [66]. While people with comorbidity have less supportive social networks than those with problematic substance use alone, such networks could still be utilised as important communication channels particularly in the vulnerable populations [66].

The current study has a number of strengths. First, the assessment of suitability of studies for the analysis, deriving the extraction rules, and the data extraction were done independently by two authors to reduce bias in the data. This issue was particularly important because some studies reported multiple estimates of ORs. Our procedure was also consistent with the data extraction from $N=7$ studies done by Moore and colleagues [10] (according to Figure six of their study) that were included in our analysis. Second, the data in both metaanalyses come from large- $N$, longitudinal surveys based on representative samples from the general population in 10 countries. Our study was more inclusive by also extracting data from small- $N$, cross-sectional studies. This approach improved the power of our analysis and allowed us to perform three separate overall analyses and subsequent subgroup analyses on more homogenous subsets of studies in contrast to Moore et al. [10] who were unable to meta-analyse the results of highly heterogeneous $N=7$ studies. While small- $N$ studies can be considered unrepresentative and of poor quality compared to large longitudinal studies, some of the former included carefully screened participants and excluded other substance users and those with other psychiatric conditions. Therefore, the samples in these smaller studies were not necessarily of 'worse quality' than those in large-scale surveys, where comorbidity with other substance use/psychiatric illness was often present and only controlled for statistically rather than by excluding such participants from further analyses. In addition, the prevalence of cannabis use and/or anxiety was low in the large longitudinal studies and, thus, the ORs in these studies were also computed based on small Ns. Third, the studies selected for the current analysis were performed in a number of countries reducing the bias in the results towards any one country and/or one research team only. Although both authors of the current study are multilingual, the search for studies was conducted in English only because most of the largest studies on the topic are conducted in the English-speaking, western world (possibly due to high costs of longitudinal studies) and/or are published in English. Consequently, even if searching in other languages, finding of non-English studies is more difficult because they are often not listed on the largest scholarly databases. Finally, a systematic assessment of publication bias with a number of tests (funnel plots in Figures 2, 3, 4 and results in Table 4) suggests that there was little evidence for such a bias in the current analysis. Specifically, our meta-analysis included studies with either positive, negative, or lack of associations between anxiety and cannabis use/cannabis use disorders rather than studies reporting only strong and positive associations.

An important limitation of the current study is the literature search strategy. While Moore et al. [10] searched a large number of databases, the current search was performed on two databases only (Table 1 ). The rationale behind this search strategy was that we expected to find the most important sources on Medline and PsycInfo databases. These two databases were also the most-relevant to the topic databases available at our institution. Our search strategy had failed to find $N=5$ published studies from Figure six in Moore et al. [10]. While this fact could be considered as clear evidence for a publication bias (or at least a poor search strategy), none of the $N=5$ studies specifically addressed anxiety and cannabis use (a combination 
of our search terms; Table 1). Instead, the titles of these studies included substance use and anxiety, cannabis use and mental health, or substance use and mental health. Furthermore, both meta-analyses had failed to electronically locate a large longitudinal study from the USA [26] even though the title contained the relevant search terms. This study was obtained from our hand searches but was omitted from Moore et al. [10] (even though it met the inclusion criteria of that study). Therefore, it is unlikely that any meta-analysis would include a complete set of studies on this topic (both published and unpublished) because, if published, they might not be listed on major scholarly databases, listed using non-specific terms only (such as substance use and mental illness), and access to all print material in all countries is hardly possible. One reasonable compromise is to conclude that our results cannot be extrapolated to the general population (of the whole world) but rather apply to countries and samples used in the current study alone. According to Table 2, if the same studies are counted only once and the latest wave is used as the total sample size/study, then the current results apply to samples drawn from approximately 112,000 cases from the general population of 10 countries.

\section{Conclusions}

The results of the current meta-analysis suggest that anxiety is positively related to cannabis use or cannabis use disorders in cohorts, not in treatment for anxiety or cannabis use disorders, drawn from the general population of 10 countries. These associations were only small in magnitude, but were observed even after controlling for confounding factors (demographics, other substance use, and/ or other psychiatric comorbidity). The prospective analysis revealed that cannabis use at baseline was positively associated with anxiety at follow-up. While the causal direction of this relationship could not be established using the available data, this result suggests that even infrequent cannabis use is associated with clinically relevant symptoms of anxiety. Therefore, an appropriate assessment of cannabis use might be necessary for an effective treatment of anxiety disorders.

\section{Additional file}

Additional file 1: Supplementary Appendix.

\footnotetext{
Abbreviations

AD: Anxiety disorder; ADAD: Adolescent Drug Abuse Diagnosis (based on Addiction Severity Index); AMD: Anxiety + depression; AP: Agoraphobia; AUDADIS: Alcohol Use Disorders and Associated Disabilities Interview Schedule; BAl: Beck Anxiety Inventory; CA: Cannabis abuse; $\mathrm{CAMH}$ : Centre for Addiction and Mental Health Monitor survey, Canada; CanDep: The Dutch Cannabis Dependence Study, Netherlands; CD: Cannabis dependence; CHDS: Christchurch Health and Development Study, New Zealand; CIDI: Composite International Diagnostic Interview; CIS-R: Clinical Interview Schedule- Revised; CSHS: Colorado Social Health Survey, USA; CUD: Cannabis
}

use disorder (abuse/harmful use and/or dependence); D + : Frequent cannabis users with dependence in CanDep study; DIS: Diagnostic Interview Schedule; DISC: Diagnostic Interview Schedule for Children;

DMHDS: Dunedin Multidisciplinary Health and Development Study, Dunedin, New Zealand; ECA: Epidemiological Catchment Area program, USA; EDSP: Early Developmental Stages of Psychopathology study, Germany; GAD: generalized anxiety disorder; GHQ-12: General Health Questionnaire (12 items); HSC: Hopkins Symptom Checklist; K-SADS: Schedule for Affective Disorders and Schizophrenia for School-Age Children; LIFE: Longitudinal Interval Follow-up Evaluation; MUSP: Mater University Study of Pregnancy, Brisbane, Australia; NCS: National Comorbidity Survey, USA; NCS-R: National Comorbidity Survey- Replication, USA; NEMESIS/NEMESIS-2: Netherlands Mental Health Survey and Incidence Study (study 1: 1996-1999 and study 2: 2007-2009); N2: NEMESIS-2 cases Netherlands; NESARC: National Epidemiological Survey on Alcohol and Related Conditions, USA; NoRMHS: The Northern Rivers Mental Health Study, New South Wales, Australia; NPMS: The British National Psychiatric Morbidity Survey, UK; NSMHWB: National Survey of Mental Health and Well-Being, all states, Australia; OAD: Overanxious disorder; OCD: Obsessive compulsive disorder; PA: Panic attacks; PD: Panic disorder; PRIME-MD: Primary Care Evaluation of Mental Disorders; PTSD: Post-traumatic stress disorder; SA: Separation anxiety; SAD: Social anxiety disorder/social phobia; SCID-I/NP: Structured Clinical Interview for DSM-IV, non-patient version; SIAS: Social Interaction Anxiety Scale; SP: Specific phobias; STAl: State-Trait Anxiety Inventory; STAI-Y: STAI for Youth; STAI-Y A: STAI state anxiety subscale; STAI-Y B: STAI trait anxiety subscale; T: Specific wave of data collection in longitudinal studies; TH2K: Teen Health 2000 Study, Houston, USA; VAHCS: Victorian Adolescent Health Cohort Study, Victoria, Australia; YASR: Young Adult Self-Report.

\section{Competing interests}

Both authors have no competing interests. There was no external funding for this study.

\section{Authors' contributions}

Both authors have equally contributed to this study: LTL conducted the systematic search, extracted data from a pilot sample of $N=17$ studies, conducted preliminary analyses on these data; KKK extracted data from all studies, conducted the final analysis, and wrote the paper; both authors agreed on the data extraction procedure, double-checked the extracted data from all $N=31$ studies, and critically revised the paper. Both authors read and approved the final manuscript.

\section{Authors' information}

Dr. Karina Kedzior completed her PhD in the clinical neurosciences at the University of Western Australia, Perth, Australia, in 2004. Her research so far has focused on the relationship between cannabis use and neurophysiological functioning (prepulse inhibition of the startle reflex) in cannabis users and schizophrenia patients. She has also investigated the validity of self-reports of cannabis and other substance use in cannabis users and schizophrenia patients, the validity of cannabis dependence diagnoses on the CIDI, and the association between cannabis use and depression. She is currently a Lecturer in Statistics and Research Methods at Jacobs University Bremen, Germany, where she teaches the traditional statistical methods (including logistic regression) and meta-analysis. She is a co-author of four other meta-analyses. Lisa Laeber completed her BA in Integrated Social and Cognitive Psychology at Jacobs University Bremen, Germany, in June 2013. Throughout her degree she studied all methods necessary for performing of the current analysis (linear and logistic regressions, meta-analysis) and completed various courses on advanced statistics in psychology (structural equation modelling, mixed methods, and an ERASMUS intensive program on quantitative approaches to psychological processes). Her bachelor thesis on the relationship between anxiety and cannabis use in $N=17$ studies submitted in April 2013 (under the supervision of Dr. Kedzior) was used as a pilot study for the purpose of this publication.

\section{Acknowledgements}

We would like to thank the authors of some primary studies included in the current analysis for additional comments and data (see Table 3, 'Data location in study' column). In addition, we thank the following colleagues at Jacobs University Bremen: Ms. Anja Kakau (Systems Librarian) for her assistance with the systematic literature search, Dr. Katja Hanke for her 
comments on the earlier version of this manuscript, and Mr. Patrick Kotzur for assistance with translation from Portuguese. Finally, we thank the following peer-reviewers for their comments on the original version of this manuscript: Profs. Low and Zvolensky, and Ms. van der Pol.

Received: 19 July 2013 Accepted: 31 March 2014

Published: 10 May 2014

\section{References}

1. Grobe T: Gesundheitsreport 2012. Techniker Krankenkasse: Hamburg, Germany; 2012.

2. Wittchen $\mathrm{H}-\mathrm{U}$, Jacobi F, Rehm J, Gustavsson A, Svensson M, Jönsson B, Olesen J, Allgulander C, Alonso J, Faravelli C, Fratiglioni L, Jennum P, Lieb R, Maercker A, van Os J, Preisig M, Salvador-Carulla L, Simon R, Steinhausen $\mathrm{H}-\mathrm{C}$ : The size and burden of mental disorders and other disorders of the brain in Europe 2010. Eur Neuropsychopharmacol 2011, 21:655-679.

3. Kessler RC, Angermeyer M, Anthony JC, DEG R, Demyttenaere K, Gasquet I, DEG G, Gluzman S, Gureje O, Haro JM, Kawakami N, Karam A, Levinson D, Medina Mora ME, Oakley Browne MA, Posada-Villa J, Stein DJ, Adley Tsang $\mathrm{CH}$, Aguilar-Gaxiola S, Alonso J, Lee S, Heeringa S, Pennell BE, Berglund P, Gruber MJ, Petukhova M, Chatterji S, Ustun TB: Lifetime prevalence and age-of-onset distributions of mental disorders in the World Health Organization's World Mental Health Survey Initiative. World Psychiatry 2007, 6:168-176.

4. Kessler RC, Avenevoli S, Costello EJ, Georgiades K, Green JG, Gruber MJ, He JP, Koretz D, McLaughlin KA, Petukhova M, Sampson NA, Zaslavsky AM, Merikangas KR: Prevalence, persistence, and sociodemographic correlates of DSM-IV disorders in the National Comorbidity Survey Replication Adolescent Supplement. Arch Gen Psychiatry 2012, 69:372-380.

5. Australian Bureau of Statistics: The National Survey of Mental Health and Wellbeing (cat. no. 4326.0). Australia: ABS; 2007

6. Degenhardt L, Chiu W-T, Sampson N, Kessler RC, Anthony JC, Angermeyer M, Bruffaerts R, de Girolamo G, Gureje O, Huang Y, Karam A, Kostyuchenko S, Lepine JP, Mora MEM, Neumark Y, Ormel JH, Pinto-Meza A, Posada-Villa J, Stein DJ, Takeshima T, Wells JE: Toward a global view of alcohol, tobacco, cannabis, and cocaine use: Findings from the WHO World Mental Health Surveys. PLoS Med 2008, 5:e141.

7. Degenhardt L, Hall W: Extent of illicit drug use and dependence, and their contribution to the global burden of disease. Lancet 2012, 379:55-70.

8. Degenhardt L, Ferrari AJ, Calabria B, Hall WD, Norman RE, MCGrath J, Flaxman AD, Engell RE, Freedman GD, Whiteford HA, Vos T: The global epidemiology and contribution of cannabis use and dependence to the global burden of disease: Results from the GBD 2010 Study. PLOS ONE 2013, 8:0076635

9. Degenhardt L, Hall WD, Lynskey M, McGrath J, McLaren J Calabria B, Whiteford H, VOS T: Should burden of disease estimates include cannabis use as a risk factor for psychosis? PLoS Med 2009, 6:29.

10. Moore THM, Zammit S, Lingford-Hughes A, Barnes TRE, Jones PB, Burke M, Lewis $G$ : Cannabis use and risk of psychotic or affective mental heath outcomes: A systematic review. Lancet 2007, 370:319-328.

11. Semple DM, McIntosh AM, Lawrie SM: Cannabis as a risk factor for psychosis: systematic review. J Psychopharmacol (Oxf) 2005, 19:187-194.

12. Large M, Sharma S, Compton MT, Slade T, Nielssen O: Cannabis use and earlier onset of psychosis: a systematic meta-analysis. Arch Gen Psychiatry 2011, 68:555-561.

13. Koskinen J, Lohonen J, Koponen H, Isohanni M, Miettunen J: Rate of cannabis use disorders in clinical samples of patients with schizophrenia: a meta-analysis. Schizophr Bull 2010, 36:1115-1130.

14. Degenhardt L, Hall W, Lynskey M: Exploring the association between cannabis use and depression. Addiction 2003, 98:1493-1504

15. Kedzior K, Martin-Iverson M: Association between severity of cannabis dependence and depression. Psychology 2010, 1:233-237.

16. Crippa JA, Zuardi AW, Martín-Santos R, Bhattacharyya S, Atakan Z, McGuire P, Fusar-Poli P: Cannabis and anxiety: A critical review of the evidence. Hum Psychopharmacol 2009, 24:515-523.

17. Manrique-Garcia E, Zammit S, Dalman C, Hemmingsson T, Allebeck P: Cannabis use and depression: a longitudinal study of a national cohort of Swedish conscripts. BMC Psychiatry 2012, 12:12-112.

18. Leweke FM, Koethe D: Cannabis and psychiatric disorders: It is not only addiction. Addict Biol $2008,13: 264-275$.
19. Peters EN, Budney AJ, Carroll KM: Clinical correlates of co-occurring cannabis and tobacco use: a systematic review. Addiction 2012, 107:1404-1417.

20. Moylan S, Jacka FN, Pasco JA, Berk M: Cigarette smoking, nicotine dependence and anxiety disorders: a systematic review of populationbased, epidemiological studies. BMC Med 2012, 10:123.

21. Moffitt TE, Harrington H, Caspi A, Kim-Cohen J, Goldberg D, Gregory AM, Poulton R: Depression and generalized anxiety disorder: cumulative and sequential comorbidity in a birth cohort followed prospectively to age 32 years. Arch Gen Psychiatry 2007, 64:651-660.

22. Smith JP, Randall CL: Anxiety and alcohol use disorders: comorbidity and treatment considerations. Alcohol Res 2012, 34:414-431.

23. Wilson N, Cadet JL: Comorbid mood, psychosis, and marijuana abuse disorders: A theoretical review. J Addict Dis 2009, 28:309-319.

24. Hall W, Lynskey M: The challenges in developing a rational cannabis policy. Curr Opin Psychiatry 2009, 22:258-262.

25. Moher D, Liberati A, Tetzlaff J, Altman D: Preferred reporting items for systematic reviews and meta-analyses: the PRISMA statement. Br Med J 2009, 339:b2535.

26. Agosti $V$, Nunes $E$, Levin F: Rates of psychiatric comorbidity among U.S. residents with lifetime cannabis dependence. Am J Drug Alcohol Abuse 2002, 28:643-652

27. Beard JR, Dietrich UC, Brooks LO, Brooks RT, Heathcote K, Kelly B: Incidence and outcomes of mental disorders in a regional population: the Northern Rivers Mental Health Study. Aust N Z J Psychiatry 2006, 40:674-682.

28. Brook J, Cohen P, Brook D: Longitudinal study of co-occurring psychiatric disorders and substance use. J Am Acad Child Adolesc Psychiatry 1998, 37:322-330

29. Brook JS, Rosen Z, Brook DW: The effect of early marijuana use on later anxiety and depressive symptoms. NYS Psychologist 2001, 13:35-40.

30. Buckner JD, Schmidt NB, Lang AR, Small JW, Schlauch RC, Lewinsohn PM: Specificity of social anxiety disorder as a risk factor for alcohol and cannabis dependence. J Psychiatr Res 2008, 42:230-239.

31. Buckner JD, Schmidt NB: Marijuana effect expectancies: Relations to social anxiety and marijuana use problems. Addict Behav 2008, 33:1477-1483.

32. Buckner JD, Joiner TE Jr, Schmidt NB, Zvolensky MJ: Daily marijuana use and suicidality: The unique impact of social anxiety. Addict Behav 2012 37:387-392

33. Cascone P, Zimmermann G, Auckenthaler B, Robert-Tissot C: Cannabis dependence in Swiss adolescents: Exploration of the role of anxiety, coping styles, and psychosocial difficulties. Swiss J Psycho/ 2011, 70:129-139.

34. Chabrol H, Ducongé E, Casas C, Roura C, Carey KB: Relations between cannabis use and dependence, motives for cannabis use and anxious, depressive and borderline symptomatology. Addict Behav 2005, 30:829-840.

35. Chabrol H, Chauchard E, Girabet J: Cannabis use and suicidal behaviours in high-school students. Addict Behav 2008, 33:152-155.

36. Cheung JTW, Mann RE, lalomiteanu A, Stoduto G, Chan V, Ala-Leppilampi K, Rehm J: Anxiety and mood disorders and cannabis use. Am J Drug Alcohol Abuse 2010, 36:118-122.

37. Cougle JR, Bonn-Miller MO, Vujanovic AA, Zvolensky MJ, Hawkins KA: Posttraumatic stress disorder and cannabis use in a nationally representative sample. Psychol Addict Behav 2011, 25:554-558.

38. Crum RM, Anthony JC: Cocaine use and other suspected risk factors for obsessive-compulsive disorder: a prospective study with data from the Epidemiologic Catchment Area surveys. Drug Alcohol Depend 1993, 31:281-295

39. Degenhardt L, Hall W, Lynskey M: The relationship between cannabis use depression and anxiety among Australian adults: Findings from the National Survey of Mental Health and Well-Being. Soc Psychiatry Psychiatr Epidemiol 2001, 36:219-227.

40. Degenhardt L, Coffey C, Carlin JB, Swift W, Moore E, Patton GC: Outcomes of occasional cannabis use in adolescence: 10-year follow-up study in Victoria, Australia. Br J Psychiatry 2010, 196:290-295.

41. Degenhardt L, Coffey C, Romaniuk H, Swift W, Carlin JB, Hall WD, Patton GC: The persistence of the association between adolescent cannabis use and common mental disorders into young adulthood. Addiction 2013, 108:124-133.

42. Fergusson DM, Lynskey MT, Horwood L: The short-term consequences of early onset cannabis use. J Abnorm Child Psychol 1996, 24:499-512. 
43. Hayatbakhsh MR, Najman JM, Jamrozik K, Mamun AA, Alati R, Bor W: Cannabis and anxiety and depression in young adults: $A$ large prospective study. J Am Acad Child Adolesc Psychiatry 2007, 46:408-417.

44. Lamers CTJ, Bechara A, Rizzo M, Ramaekers JG: Cognitive function and mood in MDMA/THC users, THC users and non-drug using controls. J Psychopharmacol (Oxf) 2006, 20:302-311.

45. Low NC, Lee SS, Johnson JG, Williams JB, Harris ES: The association between anxiety and alcohol versus cannabis abuse disorders among adolescents in primary care settings. Fam Pract 2008, 25:321-327.

46. Martins S, Gorelick D: Conditional substance abuse and dependence by diagnosis of mood or anxiety disorder or schizophrenia in the U.S. population. Drug Alcohol Depend 2011, 119:28-36.

47. McGee R, Williams S, Poulton R, Moffitt T: A longitudinal study of cannabis use and mental health from adolescence to early adulthood. Addiction 2000, 95:491-503.

48. Patton GC, Coffey C, Carlin JB, Degenhardt L, Lynskey M, Hall W: Cannabis use and mental health in young people: cohort study. Br Med J 2002, 325:1195-1198.

49. Roberts RE, Roberts $C R$, Xing $Y$ : Comorbidity of substance use disorders and other psychiatric disorders among adolescents: Evidence from an epidemiologic survey. Drug Alcohol Depend 2007, 88S:S4-S13.

50. Swift W, Coffey C, Carlin JB, Degenhardt L, Patton GC: Adolescent cannabis users at 24 years: trajectories to regular weekly use and dependence in young adulthood. Addiction 2008, 103:1361-1370.

51. van der Pol P, Liebregts $N$, de Graaf R, Ten Have M, Korf D, van den Brink W, van Laar M: Mental health differences between frequent cannabis users with and without dependence and the general population. Addiction 2013. doi:10.1111/add.12196.

52. van Laar M, van Dorsselaer S, Monshouwer K, de Graaf R: Does cannabis use predict the first incidence of mood and anxiety disorders in the adult population? Addiction 2007, 102:1251-1260.

53. Wittchen H-U, Fröhlich C, Behrendt S, Günther A, Rehm J, Zimmermann P, Lieb R, Perkonigg A: Cannabis use and cannabis use disorders and their relationship to mental disorders: A 10-year prospective-longitudinal community study in adolescents. Drug Alcohol Depend 2007, 88:S60-S70.

54. Zvolensky MJ, Bernstein A, Sachs-Ericsson N, Schmidt NB, Buckner JD, Bonn-Miller MO: Lifetime associations between cannabis, use, abuse, and dependence and panic attacks in a representative sample. J Psychiatr Res 2006, 40:477-486.

55. Zvolensky MJ, Cougle JR, Johnson KA, Bonn-Miller MO, Bernstein A: Marijuana use and panic psychopathology among a representative sample of adults. Exp Clin Psychopharmacol 2010, 18:129-134.

56. Schmidt $C$, Kohlmann $\mathrm{T}$ : When to use the odds ratio or the relative risk? Int J Public Health 2008, 53:165-167

57. Borenstein $M$, Hedges L, Higgins J, Rothstein H: Introduction to meta-analysis. John Wiley \& Sons, Ltd: Great Britain; 2009.

58. DerSimonian R, Laird N: Meta-analysis in clinical trials. Control Clin Trials 1986, 7:177-188.

59. Rosenthal J: Qualitative descriptors of strength of association and effect size. J Soc Serv Res 1996, 21:37-59.

60. Higgins J, Thompson S, Deeks J, Altman D: Measuring inconsistency in a meta-analyses. Br Med J 2003, 327:557-560.

61. Rosenthal R: The 'file drawer' problem and tolerance for null results. Psychol Bull 1979, 86:638-641.

62. Sterne J, Egger M: Funnel plots for detecting bias in meta-analysis: guidelines on choice of axis. J Clin Epidemiol 2001, 54:1046-1055.

63. Duval S, Tweedie R: A nonparametric 'trim and fill' method of accounting for publication bias in meta-analysis. J Am Statist Assoc 2000, 95:89-98.

64. Begg C, Mazumdar M: Operating characterstics of a rank correlation test for publication bias. Biometrics 1994, 50:1088-1101.

65. Egger M, Davey S, Schneider M, Minder C: Bias in meta-analysis detected by a simple graphical test. Br Med J 1997, 315:629-634.

66. Carter M, Fisher C, Isaac M: Recovery from comorbidity. SAGE Open 2013, 3:1-14.

67. McNamee R: Confounding and confounders. Occup Environ Med 2003, 60:227-234.

68. Shapiro S: Causation, bias and confounding: a hitchhiker's guide to the epidemiological galaxy. Part 1. Principles of causality in epidemiological research: time order, specification of the study base and specificity. J Fam Plann Reprod Health Care 2008, 34:83-87.

69. Solowij N, Pesa N: Cognitive abnormalities and cannabis use. Rev Bras Psiquiatr 2010, 32(Suppl 1):S31-S40.
70. Wang PS, Aguilar-Gaxiola S, Alonso J, Angermeyer MC, Borges G, Bromet EJ, Bruffaerts R, de Girolamo G, de Graaf R, Gureje O, Haro JM, Karam EG, Kessler RC, Kovess V, Lane MC, Lee S, Levinson D, Ono Y, Petukhova M, Posada-Villa J, Seedat S, Wells JE: Worldwide use of mental health services for anxiety, mood, and substance disorders: Results from 17 countries in the WHO World Mental Health (WMH) Surveys. Lancet 2007, 370:841-850.

71. van der Pol P, Liebregts $N$, de Graaf R, Korf DJ, van den Brink W, van Laar M: Predicting the transition from frequent cannabis use to cannabis dependence: a three-year prospective study. Drug Alcohol Depend 2013, 133:352-359.

72. Schulte SJ, Meier PS, Stirling J: Dual diagnosis clients' treatment satisfaction - a systematic review. BMC Psychiatry 2011, 11:64.

73. Kedzior K, Badcock J, Martin-Iverson M: Validity and consistency of self-reports regarding substance use in general research volunteers, including regular cannabis users and schizophrenia patients. Subst Use Misuse 2006, 41:743-750.

74. Large M, Smith G, Sara G, Paton M, Kedzior K, Nielssen O: Meta-analysis of self-reported substance use compared with laboratory substance assay in general adult mental health settings. Int J Meth Psych Res 2012, 21:134-148.

75. van der Pol P, Liebregts $N$, de Graaf R, Korf DJ, van den Brink W, van Laar M: Facilitators and barriers in treatment seeking for cannabis dependence. Drug Alcohol Depend 2013, 133:776-780.

doi:10.1186/1471-244X-14-136

Cite this article as: Kedzior and Laeber: A positive association between anxiety disorders and cannabis use or cannabis use disorders in the general population- a meta-analysis of 31 studies. BMC Psychiatry 2014 14:136.

\section{Submit your next manuscript to BioMed Central and take full advantage of:}

- Convenient online submission

- Thorough peer review

- No space constraints or color figure charges

- Immediate publication on acceptance

- Inclusion in PubMed, CAS, Scopus and Google Scholar

- Research which is freely available for redistribution 